\title{
Beam-beam effects at the Fermilab Tevatron: Theory
}

\author{
T. Sen, ${ }^{*}$ B. Erdelyi, M. Xiao, and V. Boocha \\ Fermi National Accelerator Laboratory, P.O. Box 500, Batavia, Illinois, 60510, USA
}

(Received 2 February 2004; published 7 April 2004)

\begin{abstract}
The Tevatron in Run II is operating with three trains of 12 bunches each. Long-range beam-beam interactions have been significant sources of beam loss and lifetime limitations of antiprotons. The dynamics due to the long-range beam-beam interactions depends on several beam parameters such as tunes, coupling, chromaticities, beam separations, intensities, and emittances. We have developed analytical tools to calculate, for example, amplitude dependent tune shifts and chromaticities, beam-beam induced coupling, and resonance driving terms. We report on these calculations and estimates of dynamic aperture and diffusion coefficients with long-term tracking. These theoretical results are compared with observations and used to predict performance at design values of beam parameters.
\end{abstract}

DOI: 10.1103/PhysRevSTAB.7.041001

PACS numbers: 29.27.Bd

\section{INTRODUCTION}

Beam-beam interactions play a major role in circular colliders. The effects of head-on interactions have a long history. However, the long-range, parasitic interactions have lately become significant in some operating colliders such as the Tevatron and CESR. Bunches in the future LHC will also be subject to these interactions. The beam dynamics issues associated with these interactions have been discussed at several workshops over the past few years; see, for example, the articles in [1-3], and references therein.

In this paper we will discuss the major theoretical aspects of the long-range interactions in the weak-strong regime as applicable to Run II in the Tevatron. Our emphasis will be on analytical calculations that allow insight into these interactions followed by numerical simulations. We will discuss specific beam-beam observations in the Tevatron only when immediately relevant to the theoretical development. Experimental studies and observations of beam-beam phenomena in the Tevatron have been published elsewhere; see, e.g., Refs. [4,5].

We start with a brief review of the Tevatron in Sec. II. Section III is devoted to the analysis of the optics of beam-beam interactions of elliptical Gaussian beams. We follow up in Sec. IV with particle tracking simulations to calculate dynamic aperture and diffusion coefficients. Section V summarizes our main results.

\section{TEVATRON: OPERATION, BEAM PARAMETERS, AND LIFETIMES}

Protons and antiprotons collide at two experimental detectors, CDF and D0. Away from the interaction points (IPs) the beams circulate on separated helical orbits within the same beam pipe. Electrostatic separators placed at several locations create these orbits. In Run

*Electronic address: tsen@fnal.gov
I there were six bunches per beam. In Run II, which started in April 2001, each beam has three trains of 12 bunches. Consequently there are 6 times as many longrange beam-beam interactions than in Run I. It was anticipated that these long-range beam-beam interactions would have a more serious impact on beam lifetime and losses. This has been borne out by observations. Beambeam observations and dedicated experiments are summarized in Refs. [4,5]. Table I contains a brief list of the important parameters.

A collider fill starts with coalesced proton bunches from the Main Injector loaded one bunch at a time onto the central orbit in the Tevatron. After all 36 proton bunches are loaded, the electrostatic separators are powered, and the protons are moved to their helical orbit. Antiprotons are loaded four bunches at a time into one of three abort gaps onto the antiproton helical orbit. For the purposes of this paper we call this stage " $\operatorname{cog} 0$ " where the four leading bunches in each train are injected. The antiproton bunches are moved longitudinally relative to the proton bunches ("cogged") by 84 rf buckets to make room for the next four bunches in the abort gap; we call this stage " $\operatorname{cog} 1 . "$ The leading eight bunches in each train are cogged again by 84 buckets to allow the injection of the last four bunches in each train. Figure 1 (taken from Ref. [6]) shows the beam configuration at injection with 36 bunches in each beam circulating.

After each train is full, which we call stage " $\operatorname{cog} 2$," the two beams are accelerated to top energy. A final cogging is done at the end of the acceleration. The optics in the interaction regions (IRs) is changed to lower the beta functions at the IPs from 1.6 to $0.35 \mathrm{~m}$. After the final step of this beta squeeze, the transverse separations at the IPs are reduced to zero with the use of the appropriate separators around the IPs. Collimators are moved in to reduce the beam halo and background in the detectors and a store begins.

In dedicated beam studies with only antiprotons injected, beam lifetimes at injection were found to be 
TABLE I. Design values of selected beam parameters in the Tevatron.

\begin{tabular}{lcc}
\hline \hline \multicolumn{1}{c}{ Parameter } & Injection & Collision \\
\hline Circumference $(\mathrm{m})$ & $(p / \bar{p})$ & \multicolumn{2}{c}{6283.187} \\
Number of bunches & \multicolumn{2}{c}{36} \\
Bunch spacing (nsec) & \multicolumn{2}{c}{396} \\
Energy (GeV) & 150 & 980 \\
$\beta^{*}$ at IP (m) & 1.6 & 0.35 \\
Normalized transverse emittance $(95 \%)(\pi \mathrm{mm}$ mrad) & \multicolumn{2}{c}{$20 / 15$} \\
Bunch intensity $\left(\times 10^{11}\right)$ & \multicolumn{2}{c}{$2.7 / 0.3$} \\
Momentum spread $(\mathrm{rms})$ & $5.5 \times 10^{-4}$ & $2.2 \times 10^{-4}$ \\
Transverse tunes & $(20.583,20.575)$ & $(20.585,20.575)$ \\
Synchrotron tune & $1.96 \times 10^{-3}$ & \multicolumn{2}{c}{$0.2 \times 10^{-4}$} \\
Beam-beam parameter & \multicolumn{2}{c}{$0.0015 / 0.0099$} \\
\hline \hline
\end{tabular}

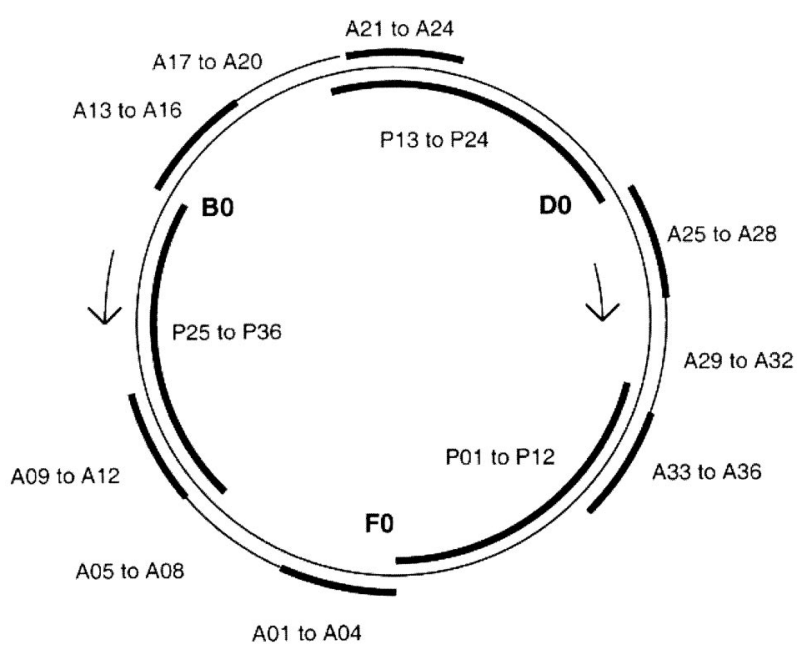

FIG. 1. From Ref. [6]. Beam configuration at injection after both beams are loaded. The proton and antiproton bunches are labeled $\mathrm{P} 01, \mathrm{P} 02, \ldots$, and $\mathrm{A} 01, \mathrm{~A} 02, \ldots$, starting from the upstream end of the bunch train so that A01 and P01 pass each other at F0. around $20 \mathrm{~h}$ and lifetimes at top energy were around $300 \mathrm{~h}$. These lifetimes are much larger at both stages than typical antiproton lifetimes in regular stores with protons present. Figure 2 shows examples of antiproton lifetimes bunch by bunch in two typical stores, one from May 2003 and another store in January 2004.

Antiproton lifetimes at injection have increased over the last year as a consequence of several improvements. These include operation with lower chromaticities following the removal of the $\mathrm{C} 0$ Lambertson and shielding the F0 Lambertson, reduced antiproton emittances due to changes in the transfer lines, and changes to the helices. The lifetime variation bunch to bunch is quite similar in the two stores shown in the figure. Antiproton lifetimes at collision over this same time period have dropped due to the increase in luminosity. The bunch to bunch variations in lifetime are quite different in the two stores since these lifetimes are more sensitive to the parameters of the proton bunches that collide with a given antiproton bunch at CDF and D0.
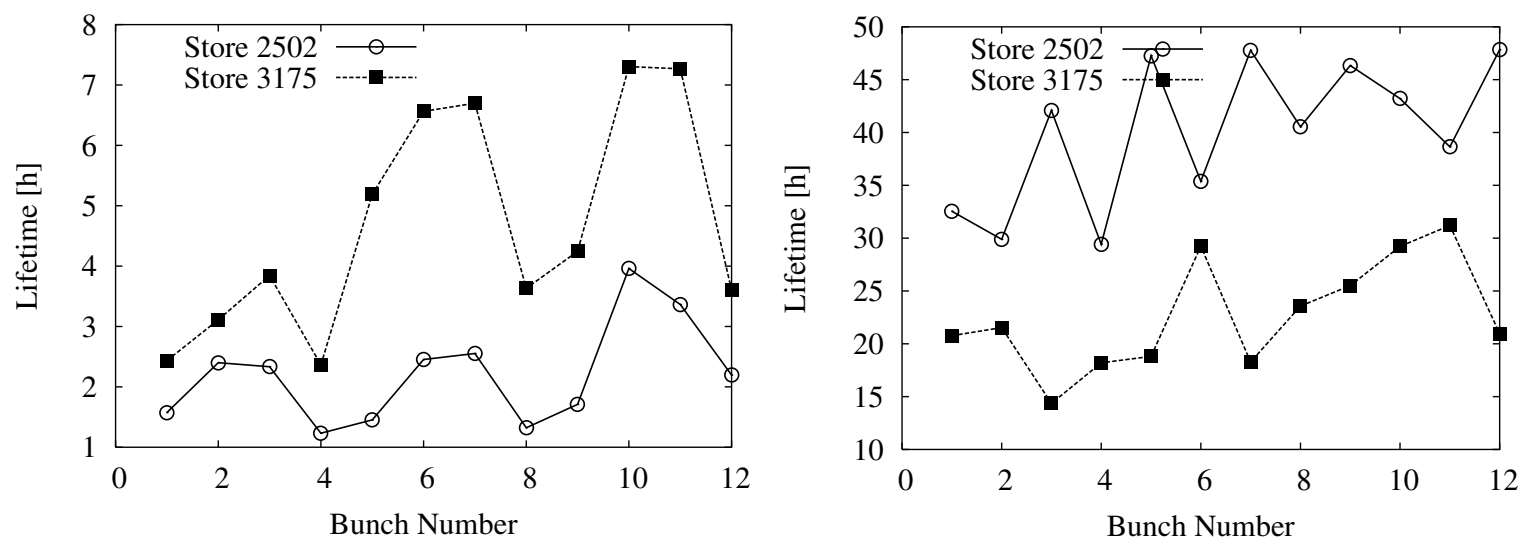

FIG. 2. Antiproton lifetimes bunch by bunch in two stores. Store 2502 occurred on May 2, 2003, while Store 3175 is a store on January 16, 2004. Left panel: At injection (150 GeV) after the second cogging. Right panel: At low beta (980 GeV) during collisions. 

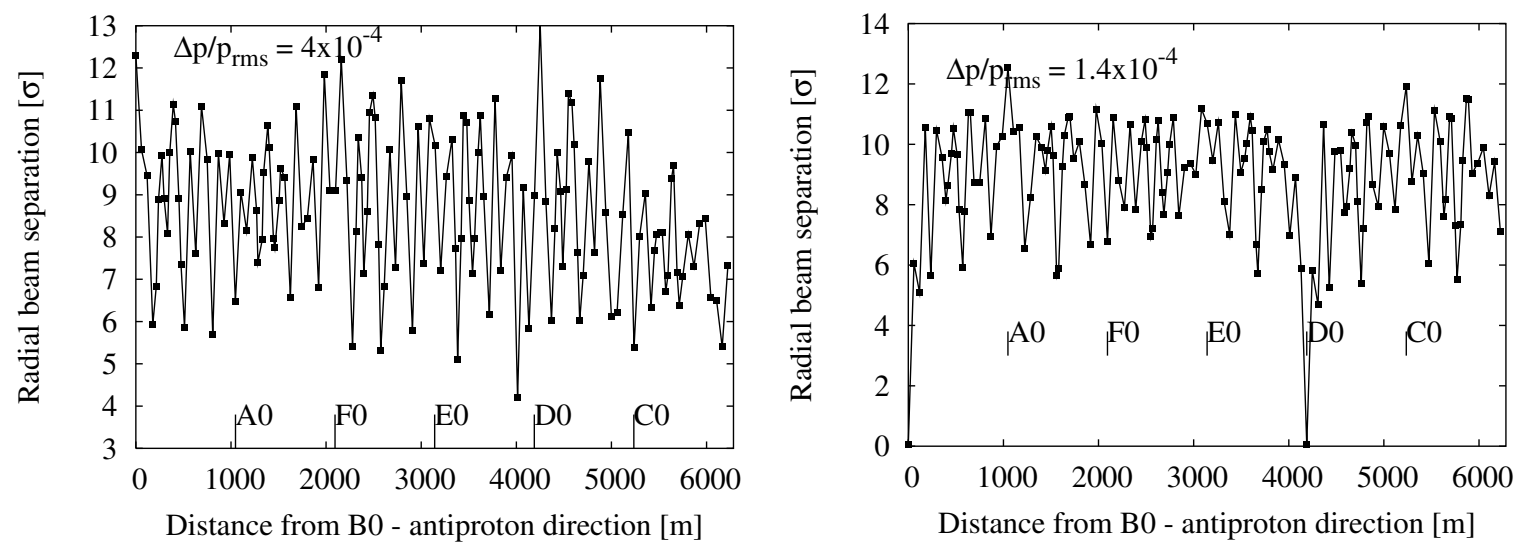

FIG. 3. Left panel: Radial beam separations at 138 interaction points around the ring. At injection $(150 \mathrm{GeV})$ after the second cogging. Right panel: At low beta $(980 \mathrm{GeV})$. The head-on collisions are at locations B0 and D0.

The configuration of long-range interactions is different at injection and collision. Each bunch experiences 72 long-range interactions at injection but at collision there are 70 long-range interactions and two head-on collisions per bunch. In total there are 138 locations around the ring where beam-beam interactions occur. The sequence of 72 interactions out of the 138 interactions is different for each bunch, hence the effects are different from bunch to bunch. The locations of these interactions and the beam separations change from injection to collision. The left plot in Fig. 3 shows the separations at all 138 interaction points in the ring after the 2 nd cogging at injection. The minimum separation is about $4 \sigma$. The right plot in this figure shows the beam separations at collision. The headon collisions occur at B0 (CDF experiment) and D0 (D0 experiment). The minimum separations $(\sim 5 \sigma)$ at the parasitic encounters occur close to the experiments.

\section{THEORETICAL OPTICS OF BEAM-BEAM INTERACTIONS}

The effects of the beam-beam force can be characterized in terms of some optical quantities, such as tune shifts, chromaticities, coupling, and resonance driving terms. We illustrate the derivation of these quantities in this section. The amplitude dependent tune-shift derivation is presented in some detail, and the derivation of the chromaticity, coupling, and resonance driving terms will be only sketched since they follow the same main ideas.

\section{A. Amplitude dependent tune shifts}

The beam-beam potential experienced by the antiprotons due to protons with Gaussian beam distributions is [7]

$$
\begin{aligned}
U= & \frac{N_{b} r_{p}}{\gamma_{p}} \int_{0}^{\infty} \frac{d q}{\left[\left(2 \sigma_{x}^{2}+q\right)\left(2 \sigma_{y}^{2}+q\right)\right]^{1 / 2}} \\
& \times\left\{1-\exp \left[-\frac{\left(x_{\beta}+L_{x}\right)^{2}}{2 \sigma_{x}^{2}+q}-\frac{\left(y_{\beta}+L_{y}\right)^{2}}{2 \sigma_{y}^{2}+q}\right]\right\},
\end{aligned}
$$

where $N_{b}$ is the number of protons per bunch, $r_{p}$ is the classical proton radius, $\gamma_{p}$ is the relativistic factor, $\left(\sigma_{x}, \sigma_{y}\right)$ are the rms proton beam sizes in the two planes, $\left(x_{\beta}, y_{\beta}\right)$ are the betatron coordinates of a test antiproton, and the separation between the two colliding bunches is $\vec{L}=\left(L_{x}, L_{y}\right)$ in the two planes, respectively (with respect to the strong beam). The expression for head-on tune shift is well known [7], and an approximation for the longrange tune shifts of round beams, which is valid in the large separation and small amplitude case, was derived in [8]. An efficient numerical integration scheme for the general case was also presented in [9].

The amplitude dependent tune shift is the advance in angle along a torus in normal form space, where a particle moves with amplitude dependent frequency. Thus, the first step of the computation is a transformation to normal form. Assuming a linearly dominated regime, it should be a very good approximation to make only a first order normal form transformation, and then take an average over the angles. The first order normal form transformation to action-angle variables is achieved by the transformation $\left(x_{\beta}, x_{\beta}^{\prime} ; y_{\beta}, y_{\beta}^{\prime}\right) \mapsto\left(J_{x}, \psi_{x} ; J_{y}, \psi_{y}\right)$, with

$$
x_{\beta}=\sqrt{2 \beta_{x} J_{x}} \cos \phi_{x}, \quad \phi_{x}=\psi_{x}+\int \frac{d s}{\beta_{x}(s)}-\nu_{x} \frac{s}{R},
$$

and similarly for the vertical plane. Assuming that the beam-beam interaction is the only perturbation to an otherwise simple harmonic motion with frequencies $\left(\nu_{x 0}, \nu_{y 0}\right)$, the Hamiltonian becomes

$$
H=\nu_{x 0} J_{x}+\nu_{y 0} J_{y}+U\left(J_{x}, \psi_{x} ; J_{y}, \psi_{y}\right) \delta\left(\theta-\theta_{c}\right),
$$


where $\delta(\theta)$ is the Dirac delta function, and $\theta=s / R$ is the independent variable. The delta function signifies that we neglect bunch length effects, and the interaction happens at a single collision point $\theta_{c}$. Introducing the tune shift as $\Delta \nu_{z}=\nu_{z}-\nu_{z 0}$, where $z=(x, y)$, from Hamilton's equations of motion we obtain that the average change in phase advance is given by the following formula:

$$
2 \pi \Delta \nu_{z}=\frac{1}{(2 \pi)^{2}} \int_{0}^{2 \pi} \int_{0}^{2 \pi} \frac{\partial U}{\partial J_{z}} d \phi_{x} d \phi_{y}
$$

The smoothness of the potential allows interchanging various integration and differentiation operations, and after performing the normal form transformation, (4) becomes (for $z=x$, and analogously for $y$ )

$$
\begin{aligned}
\Delta \nu_{x}= & C \int_{0}^{\infty} \frac{d q}{\left[\left(2 \sigma_{x}^{2}+q\right)\left(2 \sigma_{y}^{2}+q\right)\right]^{1 / 2}} \\
& \times\left[\frac{\partial}{\partial J_{x}}\left[e^{-p_{x}} I_{1}\left(J_{x}, L_{x}\right)\right]\right]\left[e^{-p_{y}} I_{2}\left(J_{y}, L_{y}\right)\right],
\end{aligned}
$$

where $C=-N_{b} r_{p} /\left[(2 \pi)^{3} \gamma_{p}\right]<0$, and

$$
\begin{aligned}
& I_{1}\left(J_{x}, L_{x}\right)=\int_{0}^{2 \pi} \exp \left(-s_{x} \cos \phi_{x}+r_{x} \sin ^{2} \phi_{x}\right) d \phi_{x}, \\
& I_{2}\left(J_{y}, L_{y}\right)=\int_{0}^{2 \pi} \exp \left(-s_{y} \cos \phi_{y}+r_{y} \sin ^{2} \phi_{y}\right) d \phi_{y} .
\end{aligned}
$$

We introduced the following shorthand notations:

$$
\begin{aligned}
& p_{x}=\frac{2 \beta_{x} J_{x}+L_{x}^{2}}{2 \sigma_{x}^{2}+q}, \quad r_{x}=\frac{2 \beta_{x} J_{x}}{2 \sigma_{x}^{2}+q}, \\
& s_{x}=\frac{2 L_{x} \sqrt{2 \beta_{x} J_{x}}}{2 \sigma_{x}^{2}+q} .
\end{aligned}
$$

Expanding the exponential in its Taylor series,

$$
e^{r_{x} \sin ^{2} \phi_{x}}=\sum_{k=0}^{\infty} \frac{r_{x}^{k} \sin ^{2 k} \phi_{x}}{k !},
$$

we obtain that

$$
I_{1}=\sum_{k=0}^{\infty} \frac{r_{x}^{k}}{k !} \int_{0}^{2 \pi} \exp \left(-s_{x} \cos \phi_{x}\right) \sin ^{2 k} \phi_{x} d \phi_{x} .
$$

If $s_{x} \neq 0$, a standard formula from the theory of Bessel functions [10] can be easily modified to give

$$
\begin{gathered}
\int_{0}^{2 \pi} \exp \left(-s_{x} \cos \phi_{x}\right) \sin ^{2 k} \phi_{x} d \phi_{x} \\
=2 \sqrt{\pi} \Gamma\left(k+\frac{1}{2}\right) I_{k}\left(s_{x}\right)\left(\frac{2}{s_{x}}\right)^{k} .
\end{gathered}
$$

Here $I_{k}\left(s_{x}\right)$ is the modified Bessel function of the first kind, and $\Gamma\left(k+\frac{1}{2}\right)$ is the gamma function. The zero amplitude tune-shift values can be obtained from this expression by taking the proper limits.

The final result is useful because the sum (10) converges for any value of $r_{x}$ and $s_{x}$, and it converges rea- sonably fast. The sum is positive definite, since every term in the sum is positive, so the formula should be numerically stable. Using the ratio test to study convergence, we obtained that (10) converges faster than $e^{r_{x}}$. Moreover, the value of the infinite sum depends on the separations $\left(L_{x}, L_{y}\right)$ but its rate of convergence does not, which makes it suitable for numerical evaluation in the long-range case. This is important, since it gives a measure of how many terms must be retained from the infinite sum. Furthermore, the maximum value of $r_{x}$ is $J_{x} / \varepsilon_{x}$, where $\varepsilon_{x}=\beta_{x} / \sigma_{x}^{2}$ is the emittance. It follows that in the infinite sum more terms need to be retained in the case of large amplitudes.

Returning to the expression for the tune shift, we obtain that

$$
\Delta \nu_{x}=4 \pi C \int_{0}^{\infty} \frac{d q}{\left[\left(2 \sigma_{x}^{2}+q\right)\left(2 \sigma_{y}^{2}+q\right)\right]^{1 / 2}} \sum_{x} \sum_{y},
$$

where

$$
\begin{aligned}
\sum_{x}=\sum_{k=0}^{\infty} \frac{\left(\frac{2 r_{x}}{s_{x}}\right)^{k}}{k !} \Gamma\left(k+\frac{1}{2}\right)[ & I_{k}\left(s_{x}\right)\left(\frac{2 k}{2 J_{x}}-\frac{2 \beta_{x}}{2 \sigma_{x}^{2}+q}\right) \\
& \left.+I_{k+1}\left(s_{x}\right) \frac{s_{x}}{2 J_{x}}\right],
\end{aligned}
$$

$$
\sum_{y}=\sum_{l=0}^{\infty} \frac{\left(\frac{2 r_{y}}{s_{y}}\right)^{l}}{l !} \Gamma\left(l+\frac{1}{2}\right) e^{-p_{y}} I_{l}\left(s_{y}\right) .
$$

The results can be expressed in a more elegant form if we change the integration variable from $q$ to $v$ by $v=$ $1 /\left[1+q /\left(2 \sigma_{x}^{2}\right)\right]$ and introduce the ratio of $\mathrm{rms}$ beam sizes $r=\sigma_{y} / \sigma_{x}$. It is also convenient to introduce dimensionless variables for the amplitudes and separations according to

$$
a_{x}=\frac{\sqrt{2 \beta_{x} J_{x}}}{\sigma_{x}}, \quad d_{x}=\frac{L_{x}}{\sigma_{x}},
$$

and similarly define $a_{y}$ and $d_{y}$. Using these notations, we obtain the following relationships:

$$
\begin{aligned}
& p_{x}=\frac{v}{2}\left(a_{x}^{2}+d_{x}^{2}\right), \quad p_{y}=f \frac{v}{2}\left(a_{y}^{2}+d_{y}^{2}\right), \\
& s_{x}=v a_{x} d_{x}, \quad s_{y}=f v a_{y} d_{y}, \quad f=\frac{r^{2}}{v\left(r^{2}-1\right)+1} .
\end{aligned}
$$

Putting everything together, the final expression for the amplitude dependent tune shift in $x$ is

$$
\Delta \nu_{x}\left(a_{x}, a_{y}, d_{x}, d_{y}, r\right)=\frac{4 \pi C}{\varepsilon_{x}} \int_{0}^{1} \frac{e^{-\left(p_{x}+p_{y}\right)}}{v\left[v\left(r^{2}-1\right)+1\right]^{1 / 2}} \sum_{x} \sum_{y} d v,
$$

where 


$$
\sum_{x}=\sum_{k=0}^{\infty} \frac{\left(\frac{a_{x}}{d_{x}}\right)^{k}}{k !} \Gamma\left(k+\frac{1}{2}\right)\left[I_{k}\left(s_{x}\right)\left(\frac{2 k}{a_{x}^{2}}-v\right)+I_{k+1}\left(s_{x}\right) \frac{s_{x}}{a_{x}^{2}}\right],
$$

$$
\sum_{y}=\sum_{l=0}^{\infty} \frac{\left(\frac{a_{y}}{d_{y}}\right)^{l}}{l !} \Gamma\left(l+\frac{1}{2}\right) I_{l}\left(s_{y}\right) .
$$

For completeness, the amplitude dependent tune shift in $y$ is similarly

$$
\Delta \nu_{y}\left(a_{x}, a_{y}, d_{x}, d_{y}, r\right)=\frac{4 \pi C}{\varepsilon_{y}} \int_{0}^{1} \frac{e^{-\left(p_{x}+p_{y}\right)}}{v\left[v\left(r^{2}-1\right)+1\right]^{1 / 2}} \sum_{x}^{\prime} \sum_{y}^{\prime} d v,
$$

where

$$
\begin{gathered}
\sum_{x}^{\prime}=\sum_{k=0}^{\infty} \frac{\left(\frac{a_{x}}{d_{x}}\right)^{k}}{k !} \Gamma\left(k+\frac{1}{2}\right) I_{k}\left(s_{x}\right), \\
\sum_{y}^{\prime}=\sum_{l=0}^{\infty} \frac{\left(\frac{a_{y}}{d_{y}}\right)^{l}}{l !} \Gamma\left(l+\frac{1}{2}\right)\left[I_{l}\left(s_{y}\right)\left(\frac{2 l}{a_{y}^{2}}-f v\right)+I_{l+1}\left(s_{y}\right) \frac{s_{y}}{a_{y}^{2}}\right] .
\end{gathered}
$$

We note that all integrals are evaluated over a finite range. In general the amplitude dependent tune shift is given by the doubly infinite series in Eqs. (17) and (20), and it can be shown that its sign is determined by the first two terms in the expansions. Indeed, in the case of vanishing amplitudes only the first two terms of the expansion survive the limit $a_{x} \rightarrow 0, a_{y} \rightarrow 0$. In the case of nonzero amplitudes the argument is based on analyzing the structure of (18). After a detailed analysis aided by the mean value theorem, the following qualitative statements can be made for the horizontal tune shift (for more details see [11]):

For small enough horizontal separation the beambeam effect is focusing at any amplitude and vertical separation. Above a certain threshold of the horizontal separation, the beam-beam interaction becomes defocusing for particles with small amplitudes and vertical separation, and focusing for particles with large horizontal amplitudes independent of the vertical separation and amplitude. The exact location of the sign change depends on the vertical separation and amplitude and beam aspect ratio.

Therefore, for given (sufficiently large) separations and given beam aspect ratio there is a boundary in the action space $\left(a_{x}, a_{y}\right)$ where the tune shifts must vanish, and the particles inside the boundary (smaller amplitudes) may have dynamically different behavior than outside the core (large amplitudes). Moreover, since the tune-shift scales like the derivative of $e^{-\left(a_{x}^{2}+d_{x}^{2}\right) / 2} I_{0}\left(a_{x} d_{x}\right)$ with respect to $a_{x}$, the tune shift starts at a negative value at zero ampli- tude, might have a minimum negative value, then cross zero and have a maximum positive value before decaying to zero at infinite amplitudes. This may result in folds in the tune footprint and could result in significant resonance widths around the folds' locations. Another type of folding may occur around the maximum value of the function $e^{-\left(a_{y}^{2}+d_{y}^{2}\right) / 2} I_{0}\left(a_{y} d_{y}\right)$ independent of the horizontal amplitude and separation. The locations of these folds are independent of the tune. If the tunes are such that the resonances occur at the folds, then we may have large resonance widths because $\Delta J_{\text {res }} \propto 1 /[\partial(\Delta \nu) / \partial J]$.

Finally, it is worthwhile noting that the cutoff value $k=\max \left[\operatorname{nint}\left(a_{x}^{2} / 2\right), 1\right]$ is a good choice for the truncation of the infinite series for practical computations. The abbreviation "nint" stands for nearest integer. In practice we find that 20 terms up to amplitudes of $6 \sigma$ suffice to achieve a relative accuracy of $10^{-6}$.

\section{Small amplitude tune shifts}

Here we will examine the tune shifts of particles at the core of a bunch. First, from (5) it is clear that $\Delta \nu_{x}$ is positive definite for head-on interactions, $\vec{L}=0$. It is clear from (17)-(19) that the tune shift depends only on the absolute value of the separations in both planes. The following results are obtained from (17) by taking analytical limits:

$$
\begin{aligned}
& \lim _{\substack{a_{x} \rightarrow 0 \\
a_{y} \rightarrow 0}} \Delta \nu_{x}=\xi_{x} \int_{0}^{1} \frac{e^{-\left(d_{x}^{2}+f d_{y}^{2} / 2\right) v}}{\sqrt{v\left(r^{2}-1\right)+1}}\left(1-d_{x}^{2} v\right) d v, \\
& \lim _{\substack{a_{x} \rightarrow 0 \\
a_{y} \rightarrow 0}} \Delta \nu_{y}=\xi_{y} \int_{0}^{1} \frac{e^{-\left(d_{x}^{2}+f d_{y}^{2} / 2\right) v}}{\sqrt{v\left(r^{2}-1\right)+1}}\left(f-f^{2} d_{y}^{2} v\right) d v,
\end{aligned}
$$

where

$$
\xi_{x, y}=\frac{N_{b} r_{p}}{4 \pi \gamma_{p} \varepsilon_{x, y}}>0
$$

is the beam-beam parameter. In the round beam case, $r=$ 1 , the integral can be done analytically to give

$$
\begin{gathered}
\lim _{\substack{a_{x} \rightarrow 0 \\
a_{y} \rightarrow 0}} \Delta \nu_{x}(r=1)=-\xi \frac{2}{d^{2}}\{ \\
\cos 2 \theta-e^{-\left(d^{2} / 2\right)}[\cos 2 \theta \\
\left.\left.+\cos ^{2} \theta d^{2}\right]\right\},
\end{gathered}
$$

where $d_{x}=d \cos \theta, d_{y}=d \sin \theta$. It is apparent from (26) that the loci of vanishing tune shifts in the case of large enough separations are close to the diagonal $\left(\theta=45^{\circ}\right)$, since the first term vanishes along the diagonal and the second term in the curly brackets is suppressed already at modest separations.

The location closest to the origin where $\Delta \nu_{x}$ changes sign is at $\left|d_{x}\right| \approx 1.5852$ for $d_{y}=0$; this is also the location of the maximum of the beam-beam force. It is worth 

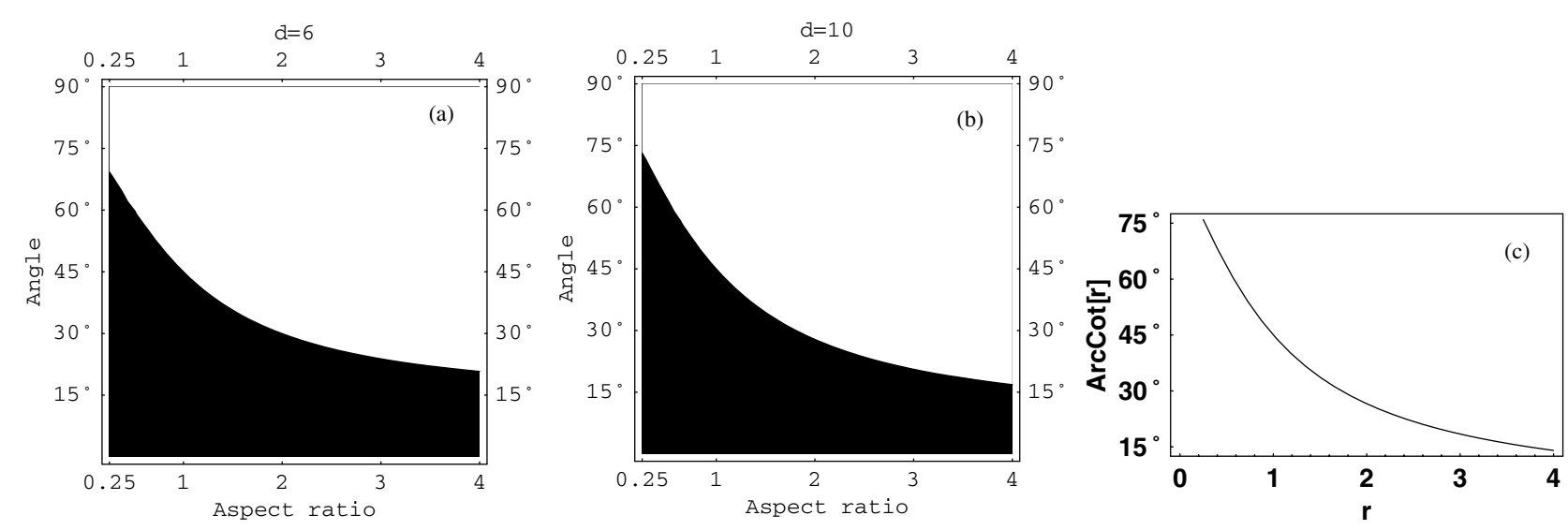

FIG. 4. Contour of vanishing tune shifts for the following cases: zero amplitudes, the separations are (a) $d=6$ (minimum separation in the Tevatron); (b) $d=10$ (average separation in the Tevatron), and the contour represents the polar angle at which the tune shifts vanish for aspect ratios in the range $[0.25,4]$. Also shown is (c) the function $\operatorname{arccot}(r)$, to which the contours apparently converge in the large separation limit.

noting that if $r>1$ then the maximum of the long-range tune shift $\max \Delta \nu_{x}<\xi$, and if $r<1$ then $\max \Delta \nu_{x}>\xi$.

The integral (23) cannot be done analytically if $r \neq 1$. In the limit of large separations, a first order perturbation calculation shows that $\lim _{d \rightarrow \infty} \theta_{\min }(\delta r)=\pi / 4-\delta r / 2$ where $\delta r=r-1$. Thus the locations of vanishing tune shift depend on the beam aspect ratio. The exact dependence can be computed by numerical integration. The global picture is revealed by the contours along which the long-range tune shift vanish, as shown in Fig. 4. Clearly, the contours tend to the graph of $\theta_{\min }=$ $\operatorname{arccot}(r)$ in the limit of large separation and show only a weak dependence on $d$. Notice that in this case

$$
\frac{L_{x}}{L_{y}}=\frac{d_{x}}{d_{y} r}=\frac{\cot \left(\theta_{\min }\right)}{r}=1
$$

Therefore if the separations are large, the tune shifts vanish at equal physical separations for any aspect ratio. This follows from the fact that the strong beam is pointlike at large distances regardless of its actual aspect ratio, so the tune-shift cancellation has the same symmetry as in the case of round beams.

Figure 5 shows the small amplitude tune shifts bunch by bunch. This pattern is repeated in the other two trains due to the threefold symmetry. The tune shifts at injection are small, of the order of 0.001 , with the vertical tune shifts much smaller. The asymmetry between the two planes occurs because the beta functions are smaller in the vertical plane at most parasitics. The spread of tune shifts is smallest in the last group of four bunches. The right plot shows the small amplitude tune shifts at collision. This variation is quite different from the bunch by bunch variation at injection. First, because the beta functions in the two planes are nearly the same, the horizontal and vertical tune shifts are comparable. Second, only the head (bunch 1) and the tail (bunch 12) of the train have tune shifts significantly different from the others, for reasons explained in Sec. IVD. Bunch 1 has a lower vertical tune shift while bunch 12 has a lower horizontal
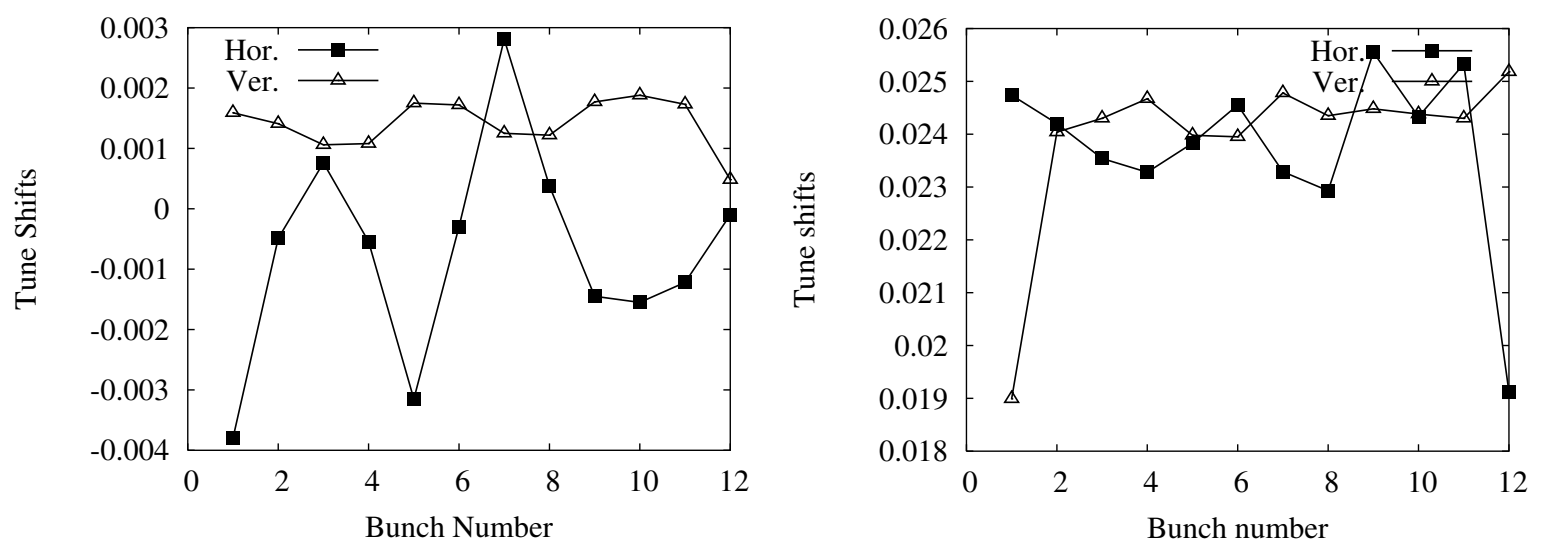

FIG. 5. Analytically calculated bunch-by-bunch small amplitude tune shifts. Left panel: at injection (cog2); right panel: at collision. 


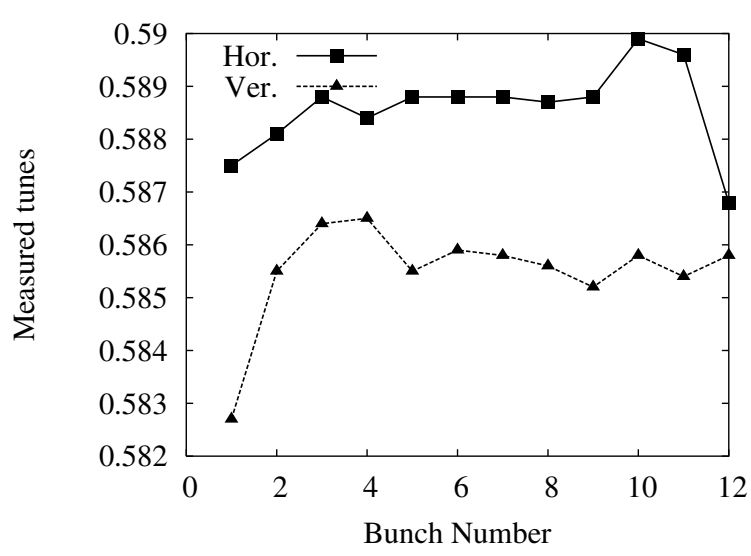

FIG. 6. Measured tunes of individual antiproton bunches at collision in a store on January 29, 2004.

tune shift. The tune shifts are about an order of magnitude larger than at injection because of the large contributions from the head-on collisions. The tune shifts calculated independently by Alexahin yielded similar results.

Very recently, reproducible measurements of individual bunch tunes at collision have been obtained from a new wideband Schottky monitor that was commissioned in 2003 [12]. Figure 6 shows the tunes of individual antiproton bunches measured several hours after the beginning of a store on January 29, 2004. These measured tunes are the tunes of the centroid motion. The tune shift of the centroid is half the value of the zero amplitude tune shift for rigid motion of the bunches [13]. The observed pattern of tune shifts reproduces several of the predicted features. In the horizontal plane, the last bunch has the smallest tune, and bunches 2 to 9 have nearly the same tunes. In the vertical plane, the first bunch has the smallest tune and the variations in the other bunches are small. The small differences from the predictions can be attributed to bunch to bunch variations in proton intensities and emittances.

\section{B. Linear chromaticity}

Just as sextupoles placed where the dispersion is nonzero change the linear chromaticity, so too do long-range interactions change the machine chromaticity when they occur at locations of nonzero dispersion. It is rather straightforward to use Eq. (17) to compute the chromaticities. We split the beam separation into two parts: one due to the closed orbits of on-momentum particles, the other due to dispersion for off-momentum particles. Denoting the dispersion (in units of rms beam size) at the location of the interaction by $\eta$, first we make the following replacements in (17):

$$
d_{x} \mapsto d_{x}+\eta_{x} \delta, \quad d_{y} \mapsto d_{y}+\eta_{y} \delta,
$$

where $\delta$ is the relative momentum or energy deviation. By definition, the linear chromaticities are given by

$$
Q_{x}^{\prime}=\left.\frac{\partial \Delta \nu_{x}}{\partial \delta}\right|_{\delta=0}, \quad Q_{y}^{\prime}=\left.\frac{\partial \Delta \nu_{y}}{\partial \delta}\right|_{\delta=0} .
$$

The derivative of the tune shift with respect to momentum deviation is cumbersome and not very illuminating. However, using the symbolic capabilities of MATHEMATICA [14], the derivative can be calculated symbolically and then evaluated numerically. To this end, the horizontal chromaticity is given by

$$
Q_{x}^{\prime}\left(a_{x}, a_{y}, d_{x}, d_{y}, r\right)=\frac{2 \pi C}{a_{x} \varepsilon_{x}} \int_{0}^{1} d v \frac{e^{-\left(p_{x}+p_{y}\right)}}{\left[v\left(r^{2}-1\right)+1\right]^{1 / 2}} \sum_{k, l=0}^{\infty}\left[\frac{\Gamma\left(k+\frac{1}{2}\right) \Gamma\left(l+\frac{1}{2}\right)}{k ! l !}\left(\frac{a_{x}}{d_{x}}\right)^{k}\left(\frac{a_{y}}{d_{y}}\right)^{l}\left[A I_{l}\left(s_{y}\right)+B I_{l+1}\left(s_{y}\right)\right]\right],
$$

where

$$
\begin{aligned}
& A=2 d_{x}\left(d_{x} \eta_{x}+d_{y} \eta_{y} f\right) v I_{k-1}\left(s_{x}\right)-a_{x}\left(3 d_{x} \eta_{x}+2 d_{y} \eta_{y} f\right) v I_{k}\left(s_{x}\right)+2 \eta_{x}\left(a_{x}^{2} v-k-1\right) I_{k+1}\left(s_{x}\right)-s_{x} \eta_{x} I_{k+2}\left(s_{x}\right), \\
& B=2 a_{y} \eta_{y} f v\left[a_{x} I_{k}\left(s_{x}\right)-d_{x} I_{k-1}\left(s_{x}\right)\right] .
\end{aligned}
$$

The vertical chromaticity can be calculated similarly. Note that the same cutoff values for $k$ and $l$ in the infinite series apply as for the tune shifts, since the convergence does not depend on the separation (i.e., dispersion).

\section{Small amplitude chromaticities}

Despite the complicated structure of (30), it can be seen that, of course, the chromaticity vanishes if there is no dispersion $\left(\eta_{x}=\eta_{y}=0\right)$, and also for head-on collisions, even for nonzero dispersions. On the other hand, for arbitrary dispersions and separations, but vanishing amplitudes, the following simplified formulas can be obtained from (23):

$$
\begin{aligned}
\lim _{\substack{\mathrm{a}_{x} \rightarrow 0 \\
a_{y} \rightarrow 0}} Q_{x}^{\prime}= & -\xi \int_{0}^{1} \frac{e^{-\left(d_{x}^{2}+f d_{y}^{2} / 2\right) v}}{\left[v\left(r^{2}-1\right)+1\right]^{1 / 2}} \\
& \times v\left[\left(v d_{x}^{2}-3\right) d_{x} \eta_{x}+\left(v d_{x}^{2}-1\right) f d_{y} \eta_{y}\right] d v, \\
\lim _{\substack{\mathrm{a}_{x} \rightarrow 0 \\
a_{y} \rightarrow 0}} Q_{y}^{\prime}= & -\xi \int_{0}^{1} \frac{e^{-\left(d_{x}^{2}+f d_{y}^{2} / 2\right) v}}{\left[v\left(r^{2}-1\right)+1\right]^{1 / 2}} \\
& \times f v\left[\left(f v d_{y}^{2}-1\right) d_{x} \eta_{x}+\left(f v d_{y}^{2}-3\right) f d_{y} \eta_{y}\right] d v
\end{aligned}
$$

In the case of round beams these integrals can be evaluated analytically. We write the general result here for the horizontal chromaticity at small amplitudes, 


$$
\begin{aligned}
q_{x}^{\prime}=\lim _{\substack{a_{x} \rightarrow 0 \\
a_{y} \rightarrow 0}} Q_{x}^{\prime}(r=1)=-\xi \frac{2}{d^{6}}\{ & 2\left(\left(d_{x}^{2}-3 d_{y}^{2}\right) d_{x} \eta_{x}-\left(d_{y}^{2}-3 d_{x}^{2}\right) d_{y} \eta_{y}\right)+e^{-\left(d^{2} / 2\right)}\left[-\left(d_{x}^{6}+d_{x}^{4}\left(2 d_{y}^{2}+1\right)\right.\right. \\
& \left.+d_{x}^{2}\left(d_{y}^{4}-2 d_{y}^{2}+2\right)-3\left(d_{y}^{2}+2\right) d_{y}^{2}\right) d_{x} \eta_{x}-\left(d_{x}^{6}+d_{x}^{4}\left(2 d_{y}^{2}+3\right)\right. \\
& \left.\left.\left.+d_{x}^{2}\left(d_{y}^{4}+2 d_{y}^{2}+6\right)-\left(d_{y}^{2}+2\right) d_{y}^{2}\right) d_{y} \eta_{y}\right]\right\} .
\end{aligned}
$$

Therefore, for large separations of the closed orbits, the zero amplitude horizontal chromaticity is

$$
\lim _{d \gg 1} q_{x}^{\prime} \approx-\xi \frac{4}{d^{3}}\left(\eta_{x} \cos 3 \theta+\eta_{y} \sin 3 \theta\right)
$$

As earlier, $\theta$ is the plane of the helix so that $d_{x}=d \cos \theta$, $d_{y}=d \sin \theta$. To obtain an order of magnitude estimate, consider a location where the horizontal dispersion is $2.5 \mathrm{~m}$ (average value over the parasitics), the beam size is $1 \mathrm{~mm}$, and the scaled separation is $d=10$. The scaled dispersion $\eta_{x}=2500$, so with $\xi \simeq 0.01$, the chromaticity contribution is $\sim 0.1$. This is a significant chromaticity when summed over all the parasitics. Notice that the chromaticity decreases faster $\left(\sim 1 / d^{3}\right)$ with separation of the closed orbits than the corresponding tune shift. Along the diagonal $\theta=\pi / 4$, for large $d$, where the tune shift approximately vanishes for round beams, the horizontal chromaticity is

$$
\lim _{d \gg 1} q_{x}^{\prime}(\theta=\pi / 4) \approx \xi \frac{4\left(\eta_{x}-\eta_{y}\right)}{\sqrt{2} d^{3}} .
$$

Therefore, it vanishes only if the dispersions are equal in the two planes which is usually only the case if both dispersions vanish. In the usual case that $\eta_{y} \ll \eta_{x}$, the horizontal chromaticity generated by the beam-beam interactions with round beams is small when the plane of the helix is either $30^{\circ}$ or $90^{\circ}$. In the more general case with nonround beams, given separation $d$ and dispersions, the angle at which the chromaticity vanishes varies with the aspect ratio. Again, it can be computed by numerical integration.

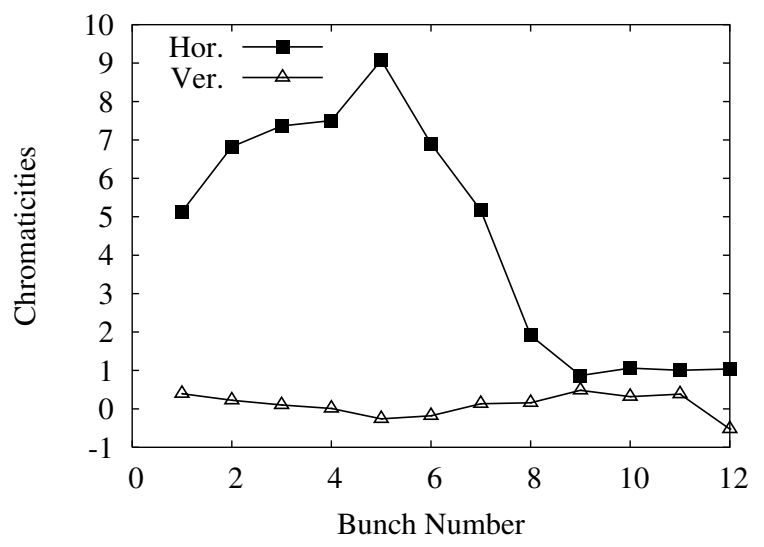

Figure 7 shows the small amplitude chromaticities of 12 antiproton bunches in a train at injection and collision. The horizontal chromaticity is much larger than the vertical because of the larger beta and dispersion functions in the horizontal plane. This additional chromaticity will enhance the synchrobetatron resonances. At injection the horizontal chromaticity of bunches 1-7 is comparable to the typical chromaticity setting of 4-8 units in the Tevatron. Bunches at the end of a train have significantly lower chromaticities; this suggests that these bunches will have better lifetimes. At collision, the beam-beam induced chromaticity is larger and spans a wider range. The bunch to bunch variation in horizontal chromaticity is similar except for bunch 10 which has a negative chromaticity. The vertical chromaticity is negative for all bunches. The machine chromaticity at collision is close to 20 units; this is sufficient to keep all bunch chromaticities positive. However this calculation does suggest that one source of bunch by bunch variations in lifetimes may be the differences in chromaticities.

\section{Minimum tune split}

In general the long-range force has a skew quadrupole component as well as higher order multipoles. These components couple the transverse motions. The global linear coupling can be parametrized by the minimum tune split which is the parameter we will calculate here. The minimum tune split is given by the amplitude of the complex term driving the difference resonance $\nu_{x}-\nu_{y}=$ $p, p \in \mathbb{Z}$. The resonance driving term is

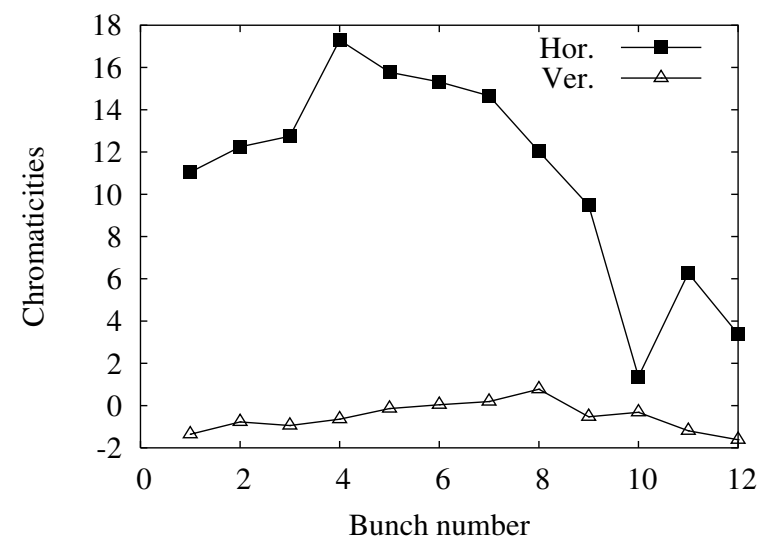

FIG. 7. Bunch by bunch small amplitude beam-beam chromaticities of antiprotons. Left panel: at injection; right panel: at collision. 


$$
\Delta \nu_{\min } e^{i \Psi}=\frac{1}{2 \pi} \oint \sqrt{\beta_{x}(s) \beta_{y}(s)} A_{x y}(s) \exp \left\{i\left[\phi_{x}(s)-\phi_{y}(s)-\left(\nu_{x}-\nu_{y}-p\right) \frac{s}{R}\right]\right\} d s,
$$

where $\phi_{x, y}(s)$ denote the phases, $R$ is the average ring radius, $A_{x y}(s)=-\partial^{2} U / \partial x \partial y$, and $\Delta \nu_{\min }$ is the minimum tune split. If there are $n$ beam-beam interaction points, with the abbreviations introduced in the previous subsection, the minimum tune split becomes

$$
\Delta \nu_{\min }=\left|-\frac{N_{b} r_{p}}{2 \pi \gamma_{p}} \sum_{j=1}^{n} \sqrt{\beta_{x, j} \beta_{y, j}} \frac{r_{j}}{\sigma_{x, j}^{2}}\left(a_{x}+d_{x, j}\right)\left(a_{y}+d_{y, j}\right) F_{j} \exp ^{\left\{i\left[\phi_{x, j}-\phi_{y, j}-\left(\nu_{x}-\nu_{y}-p\right) s_{j} / R\right]\right\}}\right| \equiv\left|\sum_{j=1}^{n} A_{j} \exp \left[i \Phi_{j}\right]\right|,
$$

where

$$
F_{j}=\int_{0}^{1} \frac{v}{\left[v\left(r_{j}^{2}-1\right)+1\right]^{3 / 2}} \exp \left[-\frac{1}{2}\left(a_{x}^{2}+d_{x, j}^{2}\right)-\frac{1}{2} \frac{r_{j}^{2} v}{v\left(r_{j}^{2}-1\right)+1}\left(a_{y}^{2}+d_{y, j}^{2}\right)\right]
$$

Since the effective skew quadrupole strength depends on the amplitude, the minimum tune split is also amplitude dependent.

We can use the second equality in Eq. (38) to write

$$
\Delta \nu_{\min }^{2}=\sum_{i} A_{i}^{2}+\sum_{\substack{i \\ i \neq j}} \sum_{j} A_{i} A_{j} \cos \left(\Phi_{i}-\Phi_{j}\right)
$$

The first single sum contributes only positive definite terms. In order for the double summation to contribute negative terms requires the phase difference $\pi / 2<\Phi_{i}-$ $\Phi_{j}<3 \pi / 2$. However the individual phases $\Phi_{i}$ are a measure of the phase slip between the transverse planes to the interaction point $i$. In regular sections of the collider such as the arcs the phase advance is nearly equal in both planes so $\Phi_{i} \sim 0$ for many interaction points. Thus minimizing the global coupling requires that the amplitude terms $A_{i}$ be individually small. More insight into these amplitude terms can be gained by looking at special cases.

\section{Small amplitude minimum tune split}

The integral for $F_{j}$ can be done analytically for round beams, $r_{j}=1$. In that case

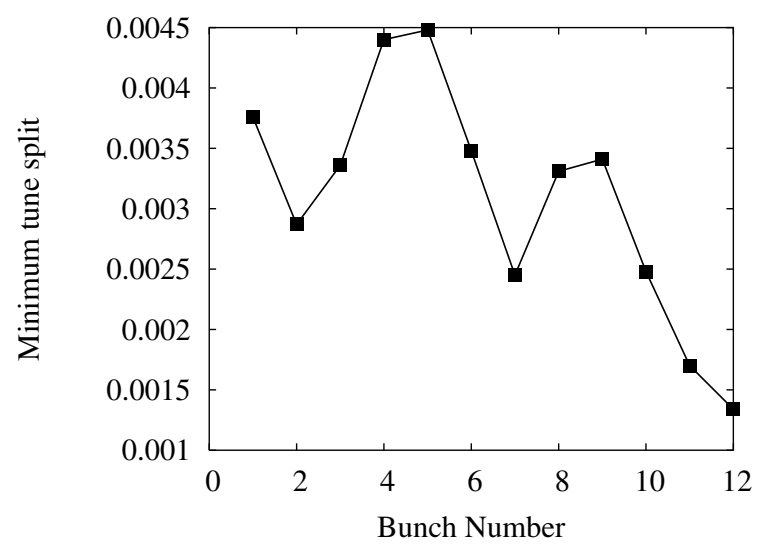

$$
F_{j}=\left(\frac{2}{T_{j}^{2}}\right)^{2}\left[1-\left(1+\frac{1}{2} T_{j}^{2}\right) \exp \left(-\frac{1}{2} T_{j}^{2}\right)\right]
$$

where $T_{j}^{2}=a_{x}^{2}+a_{y}^{2}+d_{x, j}^{2}+d_{y, j}^{2}$. At zero amplitude $a_{x}=0=a_{y}$, we can write the amplitude term as

$$
A_{j}=-\frac{N_{b} r_{p}}{2 \pi \gamma_{p}} \frac{\sqrt{\beta_{x, j} \beta_{y, j}}}{\sigma_{j}^{2}} \frac{\sin 2 \theta_{j}}{d_{j}^{2}},
$$

where $\theta_{j}$ is the angle of the plane of the helix as before. This expression shows that (i) the minimum tune split falls off as $1 / d^{2}$, similar to the tune shift, and (ii) $A_{j}$ vanishes if the plane of the helix is either horizontal or vertical. Along the diagonal $\theta=45^{\circ}$, the linear coupling is at a maximum. We noted earlier that at large distances the tune shift vanishes along the diagonal but the chromaticity in general does not vanish along the diagonal.

Using Eq. (38) we have evaluated the small amplitude minimum tune split for the 12 antiproton bunches in a train at injection and collision. Figure 8 shows the variation between the bunches. Typically, skew quadrupole circuits correct the global coupling due to the lattice to achieve a minimum tune split of about 0.003 . At injection the beam-beam induced global coupling at small amplitude is of the same order of magnitude as the lattice

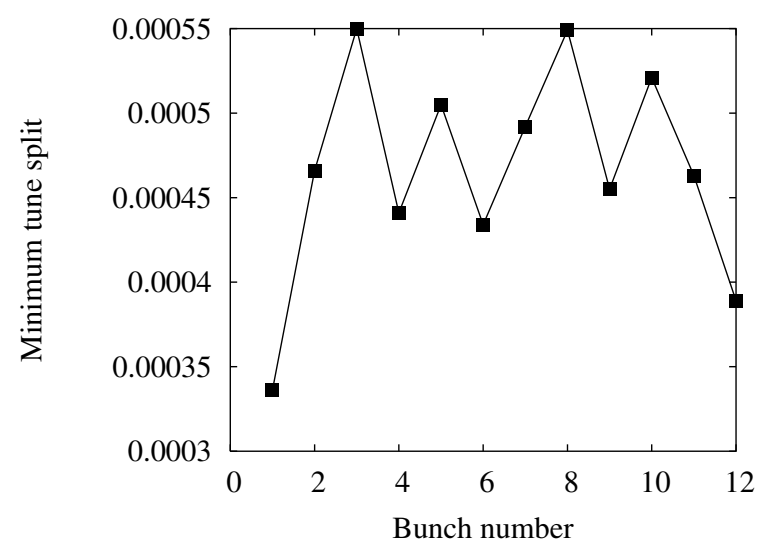

FIG. 8. Small amplitude minimum tune split due to beam-beam forces. Left panel: at injection; right panel: at collision. 
induced global coupling. Among other effects, this can make some bunches more sensitive to physical aperture limitations.

The beam-beam induced coupling at small amplitudes at collision is about an order of magnitude smaller than the machine coupling. Beam-beam induced coupling is therefore not significant at collision.

\section{Resonance driving terms}

Resonance islands are centered around the stable fixed points which can be found from the equations of motion. The widths of these resonances are found from the resonance driving terms and the detuning with amplitude, calculated in Sec. III A. Here we will calculate the resonance driving terms.

We start by splitting the phase into its periodic and nonperiodic parts, $\phi_{z}=\psi_{z}+\alpha_{z}$, where $z=(x, y), \psi_{z}$ is the canonical angle variable such that $\psi_{z}(\theta+2 \pi)=$ $\psi_{z}(\theta)+2 \pi \nu_{z}$, and

$$
\alpha_{z}(\theta)=\int_{0}^{\theta R} R \frac{d \theta^{\prime}}{\beta_{z}\left(\theta^{\prime}\right)}-\nu_{z} \theta+\phi_{0}
$$

is the periodic part $\alpha_{z}(\theta+2 \pi)=\alpha_{z}(\theta)$. The phase includes the amplitude dependent changes induced by the beam-beam force. Using the shorthand notations introduced for the tune-shift derivations, we obtain for the potential

$$
\begin{aligned}
U=\frac{N_{b} r_{p}}{\gamma_{p}} \int_{0}^{1} \frac{d v}{v\left[v\left(r^{2}-1\right)+1\right]^{1 / 2}}\left\{1-\exp \left(-p_{x}-p_{y}+\frac{r_{x}+r_{y}}{2}\right) \sum_{k_{x}, k_{y}, l_{x}, l_{y}}(-1)^{k_{x}+k_{y}+l_{x}+l_{y}} I_{k_{x}}\left(s_{x}\right) I_{k_{y}}\left(s_{y}\right) I_{l_{x}}\left(\frac{r_{x}}{2}\right) I_{l_{y}}\left(\frac{r_{y}}{2}\right)\right. \\
\left.\times \cos \left(k_{x} \phi_{x}\right) \cos \left(k_{y} \phi_{y}\right) \cos \left(2 l_{x} \phi_{x}\right) \cos \left(2 l_{y} \phi_{y}\right)\right\} .
\end{aligned}
$$

Expanding the trigonometric part into exponentials, we obtain a sum of 16 terms of the form:

$$
\exp \left\{i\left[\left( \pm k_{x} \pm 2 l_{x}\right) \phi_{x}+\left( \pm k_{y} \pm 2 l_{y}\right) \phi_{y}\right]\right\}
$$

where all 16 possible sign combinations appear in the sum. The further manipulations of each of these 16 terms is completely analogous, so we will illustrate it with only the case where everywhere the + sign is taken.

We define new indices by $m_{x}=k_{x}+2 l_{x}$ and $m_{y}=k_{y}+2 l_{y}$. The potential in terms of the action-angle coordinates can be written as $U_{++++}\left(J_{x}, \psi_{x}, J_{y}, \psi_{y}\right)=\sum_{m_{x}, m_{y}} U_{m_{x}}^{+++m_{y}} \exp \left[i\left(m_{x} \psi_{x}+m_{y} \psi_{y}\right)\right]$. Since $U_{m_{x}, m_{y}}^{++++}$is periodic in $\theta$, it can be expanded into a Fourier series,

$$
U_{++++}=\sum_{m_{x}, m_{y}, p} U_{m_{x}, m_{y}, p}^{+++} \exp \left[i\left(m_{x} \psi_{x}+m_{y} \psi_{y}-p \theta\right)\right],
$$

where

$$
U_{m_{x}, m_{y}, p}^{++++}=\frac{1}{2 \pi} \int U_{m_{x}, m_{y}}^{++++} \exp (i p \theta) d \theta .
$$

For several infinitesimal beam-beam kicks, the integral reduces to a sum over the kicks, and the resonance driving terms become

$U_{m_{x}, m_{y}, p}^{++++}=\frac{1}{16} \frac{r_{p}}{2 \pi \gamma_{p}}(-1)^{m_{x}+m_{y}-1} \sum_{n} N_{b, n} \int_{0}^{1} \frac{d v}{v\left[v\left(r^{2}-1\right)+1\right]^{1 / 2}} \exp \left(-t_{x, n}-t_{y, n}\right) W_{x, n} W_{y, n} \exp \left[i\left(m_{x} \alpha_{x, n}+m_{y} \alpha_{y, n}+p \theta_{n}\right)\right]$,

where $n$ runs over the beam-beam kicks, and

$$
\begin{gathered}
t_{x}=\frac{v}{2} d_{x}\left(d_{x}-2 a_{x}\right), \quad t_{y}=f \frac{v}{2} d_{y}\left(d_{y}-2 a_{y}\right), \\
W_{x}=\sum_{l_{x}}(-1)^{l_{x}}\left[\exp \left(-s_{x}\right) I_{m_{x}-2 l_{x}}\left(s_{x}\right)\right]\left[\exp \left(-\frac{r_{x}}{2}\right) I_{l_{x}}\left(\frac{r_{x}}{2}\right)\right], \\
W_{y}=\sum_{l_{y}}(-1)^{l_{y}}\left[\exp \left(-s_{y}\right) I_{m_{y}-2 l_{y}}\left(s_{y}\right)\right]\left[\exp \left(-\frac{r_{y}}{2}\right) I_{l_{y}}\left(\frac{r_{y}}{2}\right)\right] .
\end{gathered}
$$

The differences among the 16 different cases will show up in the definition of the $W_{x}$ and $W_{y}$ through the indices of the Bessel functions. It is easy to see that all the $W$ s for the different cases can be brought to the form of (50) and (51), respectively. Using the symmetry relation $I_{-n}(z)=$ $I_{n}(z)$, and the change in the summation from $l_{x} \mapsto-l_{x}$ if necessary, it follows that

$$
\sum_{l_{x}}(-1)^{l_{x}} I_{ \pm m_{x} \pm 2 l_{x}}\left(s_{x}\right) I_{l_{x}}\left(\frac{r_{x}}{2}\right)=\sum_{l_{x}}(-1)^{l_{x}} I_{m_{x}-2 l_{x}}\left(s_{x}\right) I_{l_{x}}\left(\frac{r_{x}}{2}\right)
$$

Therefore, the total resonance driving term is just 

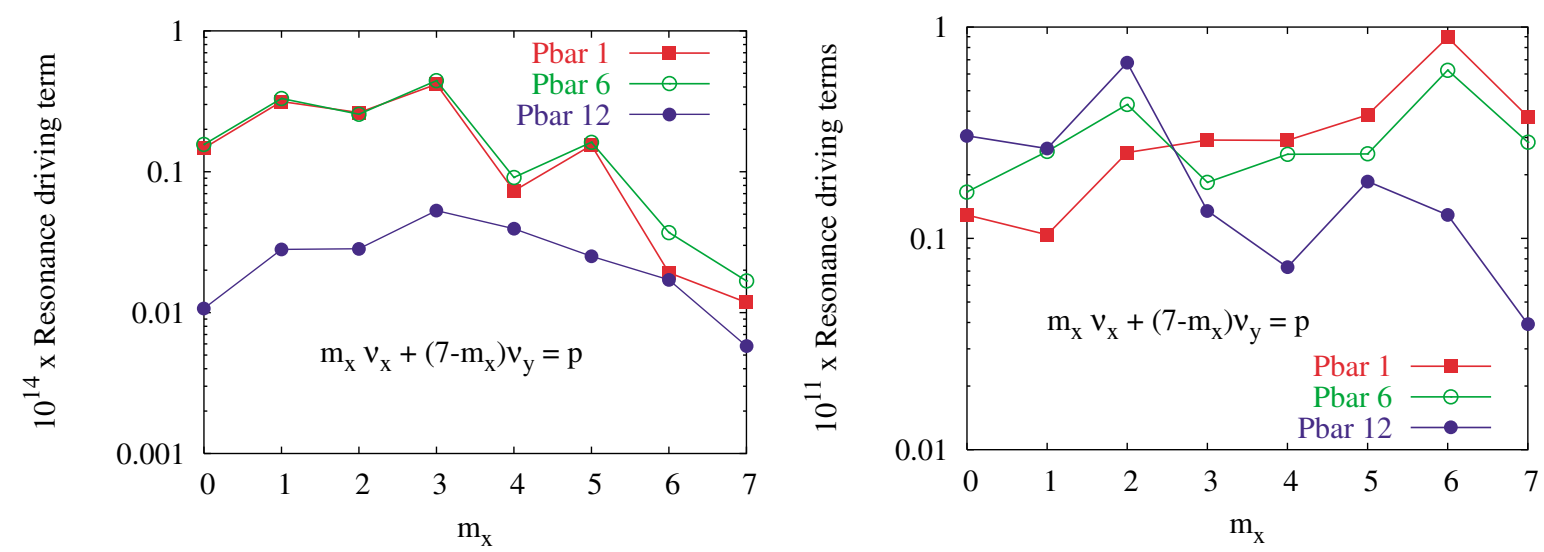

FIG. 9. (Color) Seventh order resonance driving terms on antiproton bunches 1, 6, and 12 at a $6 \sigma$ amplitude. Left panel: at injection; right panel: at collision.

16 times (48), $U_{m_{x}, m_{y}, p}=16 U_{m_{x}, m_{y}, p}^{+++}$and the complete beam-beam potential is

$$
\begin{aligned}
U\left(J_{x}, \psi_{x}, J_{y}, \psi_{y}\right)= & \operatorname{Re} \sum_{m_{x}, m_{y}, p} U_{m_{x}, m_{y}, p} \\
& \times \exp \left[i\left(m_{x} \psi_{x}+m_{y} \psi_{y}-p \theta\right)\right] .
\end{aligned}
$$

The functions $W_{x}, W_{y}$ involve infinite sums over the scaled Bessel functions $e^{-z} I_{n}(z)$. These scaled Bessel functions decrease rapidly with increasing order $n$, albeit more slowly for larger arguments. For very large arguments, keeping the leading terms in the asymptotic expansion, we have $\lim _{z \rightarrow \infty} e^{-z} I_{n}(z)=1 / \sqrt{2 \pi z}$, independent of the order $n$. Since the terms in the sums for $W_{x}, W_{y}$ alternate in sign, it implies, for example, that $\lim _{a_{x} d_{x} \rightarrow \infty} W_{x}=0=\lim _{a_{y} d_{y} \rightarrow \infty} W_{y}$. Thus we find that even at large amplitudes $\sim 6 \sigma$, about 40 terms in the summations are sufficient to achieve a relative error of $10^{-6}$.

\section{Resonance driving terms at injection and collision}

Beam lifetimes in the Tevatron are observed to drop in the vicinity of seventh order resonances. We have used Eq. (48) to calculate the strength of the beam-beam driven seventh order resonances. Figure 9 shows the resonance driving terms for three selected bunches at injection and collision. The resonances are evaluated at a $6 \sigma$ amplitude. At injection several resonances are about an order of magnitude weaker for bunch 12 compared to bunches 1 and 6 . Thus tune shifts, chromaticities, coupling and resonance strengths all suggest that antiproton lifetimes improve towards the end of a train. At collision, some resonances, e.g., $7 \nu_{y}, \nu_{x}+6 \nu_{y}, 2 \nu_{x}+5 \nu_{y}$ resonances are stronger for bunch 12 than for the other two bunches while the remaining resonances are weaker. The correlation between bunch number and lifetime is less obvious in this case. Most of the observed variations in antiproton lifetime at collision can be related to var- iations in proton bunch intensities. We note that the strengths of the twelfth order resonances driven by the head-on interactions are much weaker than the seventh order resonances driven by the long-range interactions.

Analysis of the resonance strengths leads to insight into several aspects of the beam-beam interactions. As an example, Fig. 10 shows the variation of the strengths with transverse amplitude for antiproton bunch 6 at top energy. The resonance strengths are orders of magnitude smaller at $1 \sigma$ compared to values at $6 \sigma$. Thus particles in the tails will be driven to larger amplitudes while particles in the core will be relatively unaffected. These resonance strengths have also been used to analyze the impact of changing the helix size, changes in the proton emittance, and operation with $18 \times 18$ bunches. In the last case the luminosity can be kept constant if the antiproton bunch intensity is doubled. At injection the resonance strengths are found to be significantly weaker (up to 4 times) which suggests that the antiproton lifetime

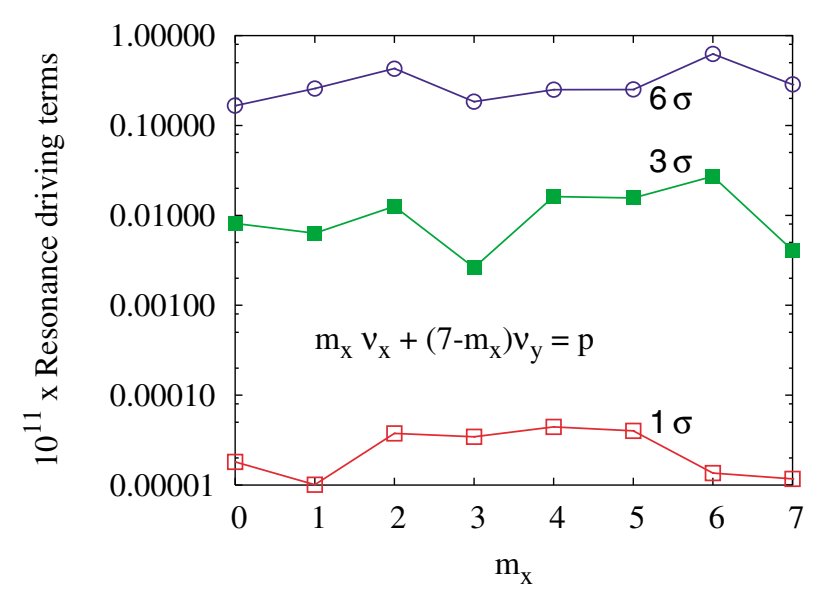

FIG. 10. (Color) The 7th order resonance driving terms at different amplitudes for antiproton bunch 6 at $980 \mathrm{GeV}$. The resonance strengths are very weak in the core of the beam and much stronger in the tails. 
would improve with an $18 \times 18$ operation. The drawback with fewer bunches is that the number of $p-\bar{p}$ interactions per crossing in the detectors increases during collisions so this would not be a feasible option above luminosities of $10^{32} \mathrm{~cm}^{-2} \mathrm{~s}^{-1}$.

\section{PARTICLE TRACKING SIMULATIONS}

Analytical calculations have shown how the beambeam interactions influence beam parameters such as orbits, tunes, coupling, chromaticity, and resonance widths. These can be used to optimize the helices and reduce the impact of the beam-beam forces. However one cannot extract dynamical quantities such as the dynamic aperture, lifetime, or emittance growth from these static parameters. Numerical simulations can be used to follow the time evolution, albeit over limited times. In this section we report on simulations to calculate the dynamic aperture and diffusion coefficients to complement the analytical calculations.

Dynamic aperture calculations were done using two different codes, MAD [15] and SIXTRACK [16]. Typically about 200 particles are followed for about $10^{5}$ to $10^{6}$ turns ( 2 to $20 \mathrm{~s}$ in the Tevatron). These codes cannot however be used for lifetime calculations which are computationally more demanding. We have developed another code BBSIM for this purpose as well as for calculations of diffusion coefficients and emittance growth. This is a parallelized code that can follow many more particles for longer periods of time, e.g., $10^{4}$ particles for $10^{6}-10^{7}$ turns in lifetime calculations. This simulation model at present includes only the linear maps between the beambeam interactions and the nonlinear beam-beam interactions in order to limit the computing time. Similar programs for Tevatron beam-beam lifetime calculations have been developed at LBNL [17] and at SLAC [18].

\section{A. Lattice model}

The Tevatron lattice model used in the dynamic aperture codes is based on the design lattice and machine settings. The nonlinearities in the model include the measured multipoles in the magnets, the chromaticity and feed-down sextupoles, together with the beambeam interactions. A systematic skew quadrupole component of $a_{1}=1$ unit in the arc dipoles is included together with the skew quadrupole circuits that correct the minimum tune split to 0.001 . The measured misalignments, mainly the rolls in dipoles and the quadrupoles, are included, and the rms closed orbit is corrected to within $0.2 \mathrm{~mm}$.

\section{B. Modeling of beam-beam effects}

There are two head-on interaction points and 70 longrange interaction points around the ring at collision, and 72 long-range interaction points at the other stages of the operational cycle. Proton bunch intensities are about 10 times larger than the antiproton bunch intensities, so beam-beam effects have largely been important for the antiprotons. Therefore, we calculate only the weak-strong beam-beam effects of the strong bunches (protons) on the weak bunches (antiprotons).

For head-on interaction points, bunch length effects are taken into account because the beta function at the IP, $\beta^{*}=35 \mathrm{~cm}$, is about the same as the design bunch length of $37 \mathrm{~cm}$. It was shown in Ref. [19] that the phase averaging effect [20] over the bunch length is significant in the Tevatron and can reduce the transverse resonance widths by up to 2 orders of magnitude. In our simulations, the strong bunch is sliced into nine disks of charge of equal length. The transverse size of each disk is different because of the rapid change in the beta function. The longitudinal charge density of each disk falls off as a Gaussian from the center of the bunch. Particles in the weak bunch are subject to impulsive kicks from the center of the disk followed by a drift to the center of the next disk so that the phase propagation between the slices is also taken into account.

Long-range interactions are modeled by impulsive kicks applied to the weak beam since the beta functions at the locations of these interactions are much larger than the bunch length and the phase change over the length of the bunch is negligible.

\section{Simulations at injection}

Machine nonlinearities have a strong influence on beam lifetime at injection. For example, lifetimes of coalesced proton bunches are 5-6 h on the central orbit but drop to $1-2 \mathrm{~h}$ when the protons are moved to their helical orbit. Uncoalesced proton bunches, which have a smaller momentum spread, have larger lifetimes than coalesced bunches but their lifetime is also smaller on the helix compared to their lifetime on the central orbit. These observations are qualitatively reproduced in our dynamic aperture calculations. Figure 11 shows the dynamic aperture (DA) in units of the beam size $\sigma$ of protons (without beam-beam effects) after $10^{5}$ turns. The DA of protons with $(\Delta p / p)_{\mathrm{rms}}=10^{-4}$ is $10 \sigma$ on the central orbit and it drops to $8.5 \sigma$ on the helix. Protons with $(\Delta p / p)_{\mathrm{rms}}=5 \times 10^{-4}$, typical of coalesced bunches, have a DA of $8.5 \sigma$ on the central orbit and a DA $\leq 6 \sigma$ on the helix. The physical aperture on the helix at injection is estimated to be about $6 \sigma$.

Our simulations for antiprotons do include the beambeam forces. At injection these beam-beam forces change with each cogging. Figure 12 shows the beam-beam separations of antiproton bunch 1 at the three different cogging stages.

As an example we consider the tune footprints shown in Fig. 13 for amplitudes between $0-6 \sigma$. The tune spreads from the beam-beam forces are of the order of 0.001 at 


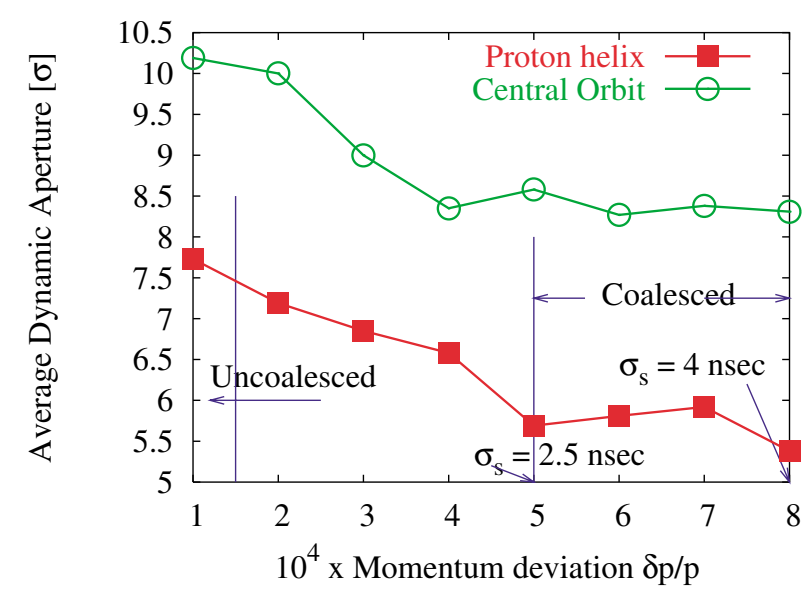

FIG. 11. (Color) Dynamic aperture of protons at injection (without beam-beam effects).

$\operatorname{cog} 0$ and $\operatorname{cog} 2$ but the footprint is smaller at $\operatorname{cog} 1$. These plots show that relatively small changes in beam separations can have a dramatic influence on the tune footprint. Folds in the footprint (where the slope of the tune with amplitude changes sign) can be seen at each stage but they occur at different amplitudes. If the amplitude at the fold coincides with the location of the resonances, the resonance width is very large. It turns out however at injection that the tune footprint is dominated by the machine nonlinearities. The tune footprint is much larger
( $\sim 0.005$ in the horizontal plane) when machine nonlinearities are included and there are no indications of folds. Consequently the tune footprints at all cogging stages are virtually the same.

\section{Dynamic aperture of antiprotons}

The helical orbits of antiprotons and protons are almost symmetrical about the central orbit. Thus we see a similar drop in DA of antiprotons in moving from the central orbit to the antiproton helix when only machine nonlinearities are considered. DA calculations with beam-beam effects have been performed for different values of several parameters including proton intensities, different bunches, cogging stages, momentum spread, chromaticities, proton emittances, etc. Here we report on a select sample of these. The left plot in Fig. 14 shows the average dynamic aperture after $10^{6}$ turns as a function of the proton bunch intensity, obtained from MAD and SIXTRACK. The averaging is done over several angles in physical space. The one-sided error bars represent the minimum dynamic aperture at each intensity. The typical range of variation between the average and the minimum DA is about $1 \sigma$. The average DA after $10^{6}$ turns without beam-beam effects is $\sim 6 \sigma$. From this plot we conclude that (i) beam-beam effects at the proton intensities of interest reduce the DA by $\sim 2 \sigma$, and (ii) the DA is nearly independent of the proton intensities over this range.

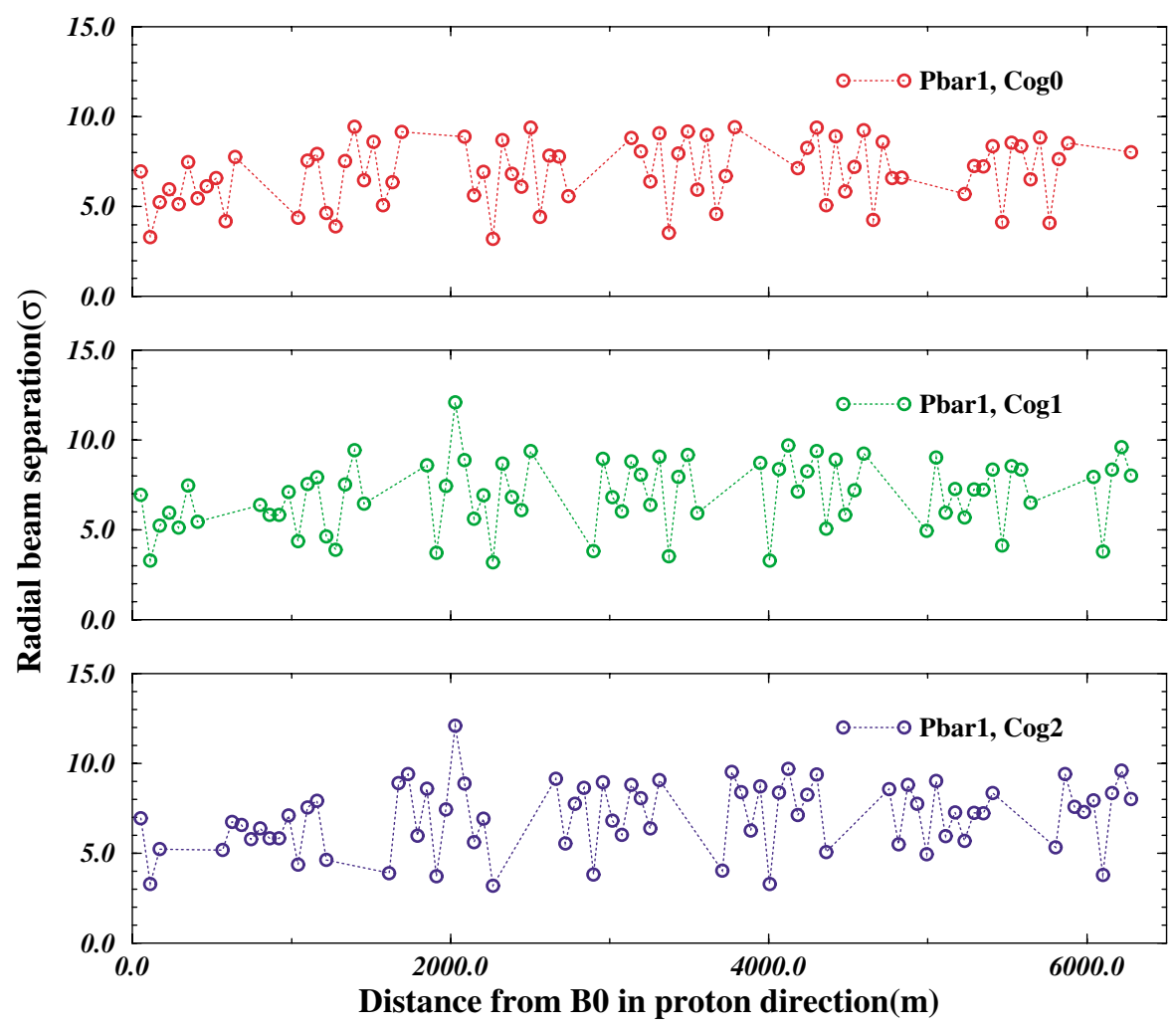

FIG. 12. (Color) Beam-beam separations of antiproton bunch 1 at the three cogging stages. 

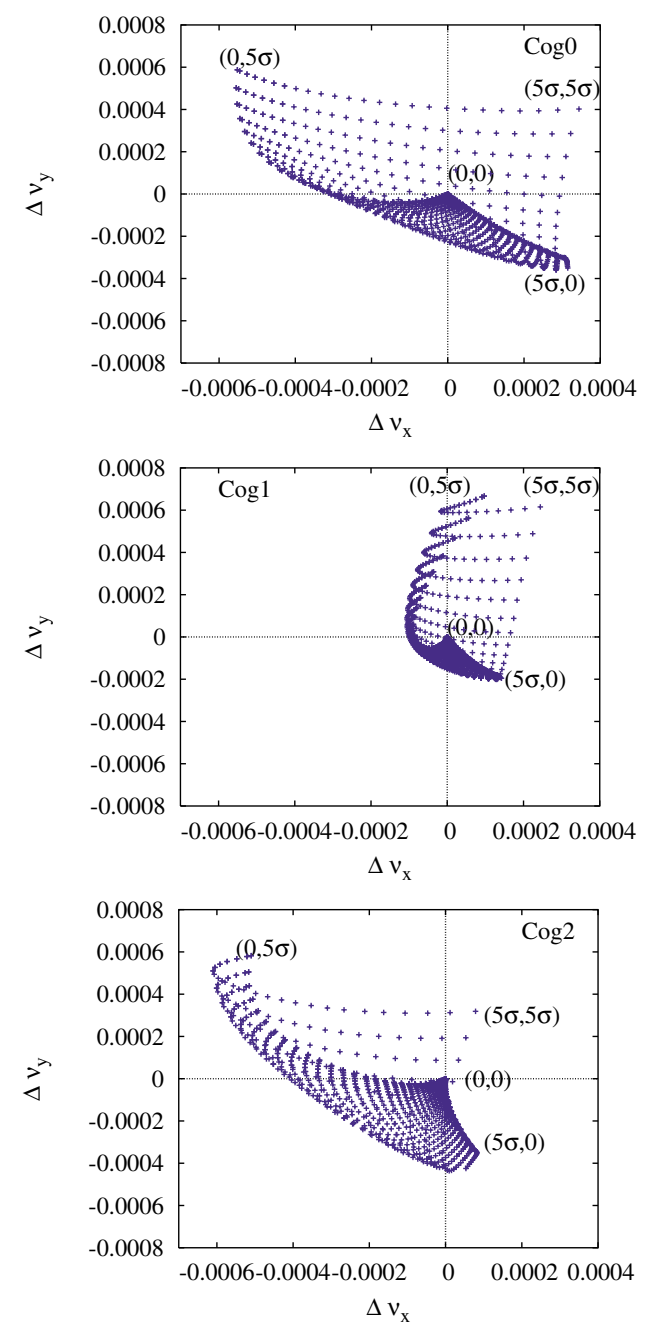

FIG. 13. (Color) Footprint of antiproton bunch 1 at the three cogging stages.

We were able to measure the DA in stores where we observed a reduction in the emittance after the antiprotons were injected. The emittances of the first four bunches were measured with flying wires 10 times during

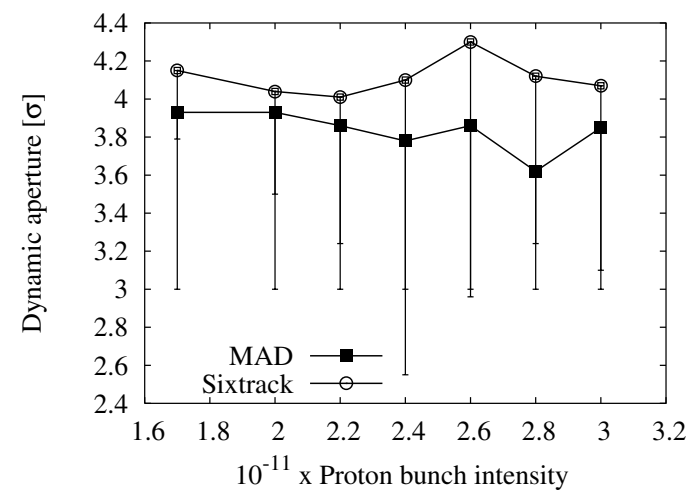

the 15-20 min these bunches circulated in the Tevatron before acceleration. The observed drop in emittance implies that a significant fraction of particles were initially outside the dynamic aperture and were lost. The asymptotic emittance is therefore an experimental measure of the dynamic aperture. Taking into account the reduction in both the horizontal and vertical planes, the measured dynamic aperture is shown in the right plot of Fig. 14. The error bars represent the statistical fluctuations (about $10 \%$ ) over the ten stores from which the data were obtained. These errors are mainly due to the changes in beam parameters such as proton intensities and machine conditions. The measured range of DAs is within the range of the calculated DA values shown in the left plot. The intensity reduction expected from assuming that the beam is contained within the estimated dynamic aperture was also in good agreement with the observed intensity loss.

Experimental checks of our other DA simulations require dedicated beam time. This beam time is at a premium during collider operation. Instead we have to rely on the very indirect relationship between the dynamic aperture and beam lifetime. Calculations of the DA at different cogging stages reveal that the average DAs are about the same at all three stages. This is not inconsistent with observations which show that the lifetime of the first group of bunches does not change significantly between stages. Lifetime measurements at each stage are however limited by insufficient statistics. On average the beams stay in each stage for 3-4 min, so often there is insufficient data from the intensity monitors for reliable lifetime measurements.

\section{Diffusion coefficients}

We have calculated diffusion coefficients at different amplitudes using the code BBSIM which includes only the nonlinearities from the beam-beam interactions. Briefly, the diffusion coefficients are calculated as follows. The

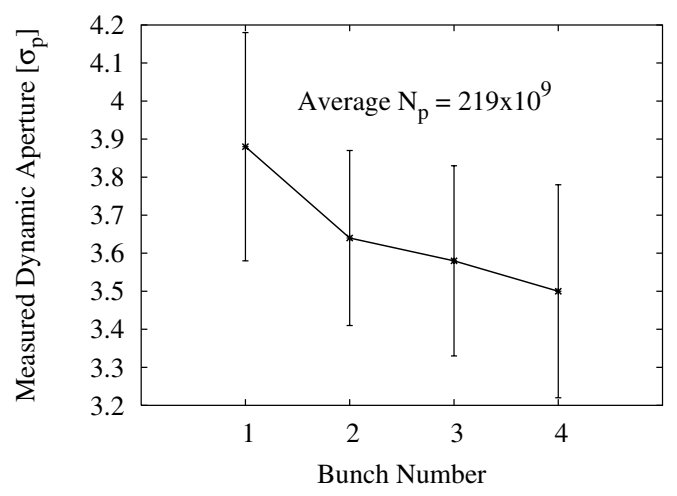

FIG. 14. Left panel: Dynamic aperture from simulations of antiproton bunch 1 at $150 \mathrm{GeV}$ after $10^{6}$ turns. Right panel: Measured dynamic aperture of antiproton bunch 1 at $150 \mathrm{GeV}$. The data were obtained from 10 stores in April 2003 when the chromaticities were higher than present values. See the text for a discussion of the error bars. 
horizontal diffusion coefficient at an amplitude $A$ after $N$ turns is

$$
D J_{x}(A)=\frac{1}{N}\left\langle\Delta\left[\operatorname{Var} J_{x}(A)\right]\right\rangle,
$$

where $\Delta\left[\operatorname{Var} J_{x}(A)\right]$ is the change in the variance of the horizontal action. The double average $\langle\langle\rangle$ signifies two averages: the action at each turn is first averaged over 100 particles placed at each amplitude and then a second average is taken every 1000 turns (about two synchrotron periods) to eliminate short term amplitude beating from phase space distortions. The variance of this averaged action is calculated. We note here that diffusion coefficients relevant to the $\mathrm{LHC}$ were calculated analytically in Ref. [21] for motion in 1 degree of freedom with round beams.

As an example, Fig. 15 shows the diffusion coefficients at a $5 \sigma$ amplitude from the individual parasitics for antiproton bunch 1 as a function of the beam separation. Particles were tracked for $10^{6}$ turns. Longer tracking did not change the coefficients much. We observe that two parasitics with small separations of $5.2 \sigma$ and $6.4 \sigma$ have the largest diffusion coefficients but the parasitic with the smallest separation has a very low diffusion coefficient. This shows that merely increasing the minimum separation is not enough but larger separations at several parasitics with small separations is required. The diffusion in the vertical plane is on average an order of magnitude larger than in the horizontal plane. The diffusion coefficients do not scale linearly with the number of parasitics. Instead with all 72 parasitics included, the average diffusion coefficients are about 6 to 7 orders of magnitude larger. This is to be expected since diffusion is very sensitive to the phase space structure created by the web of resonances from the nonlinearities.

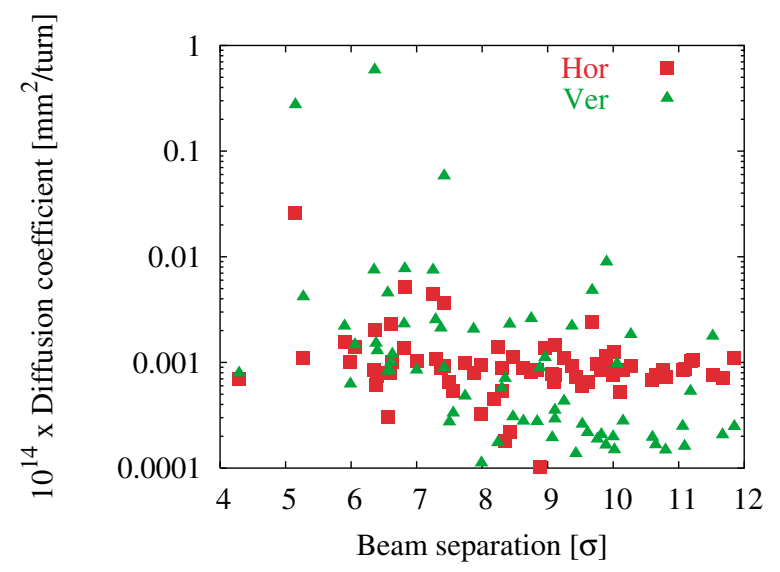

FIG. 15. (Color) Diffusion coefficients for antiproton bunch 1 at injection $(150 \mathrm{GeV})$ from individual parasitics plotted as a function of the separation at the parasitic.

\section{Simulations at collision}

At collision the beta functions at the two IPs (B0 and D0) are reduced to $35 \mathrm{~cm}$. As a result of the beta squeeze, the beta functions in the IR quadrupoles increase to $\sim 1200 \mathrm{~m}$ compared to values of $\sim 100 \mathrm{~m}$ in the arcs. The nonlinearities in these IR quadrupoles are therefore the dominant machine nonlinearities at collision.

The helical separations also change at collision. The beam separations at all the beam-beam interactions were seen earlier in Fig. 3. The beta functions at the four parasitics nearest to the IPs are the largest, the separations are small, and consequently the tune shifts and the resonance driving terms contain the largest contributions from these parasitics.

It is useful to first discuss the dynamics with only the machine nonlinearities. The tune spread between amplitudes $0-6 \sigma$ from these nonlinearities is fairly small, about 0.0006 . This is completely swamped by the tune spread of antiprotons from just the head-on interactions alone, about 0.02 at present proton intensities. The average DA after $10^{6}$ turns on the antiproton helix from the machine nonlinearities is about $12 \sigma$. The single beam DA is thus larger than the physical aperture $(\sim 6 \sigma)$ set by the primary collimators during a store.

Lifetimes of single beams at top energy are observed to be $\sim 300 \mathrm{~h}$ during machine studies. These lifetimes are close to expected values taking into account scattering off the residual gas and intrabeam scattering. Hence the IR quadrupoles or other machine elements do not by themselves limit beam lifetimes under normal conditions. When the beams collide, the lifetime is much lower mainly due to $p-\bar{p}$ interactions at the IPs. The antiproton lifetime from these interactions is

$$
\tau_{\mathcal{L}}(\bar{p})=\frac{N_{T ; \bar{p}}}{2 \mathcal{L} \Sigma_{p \bar{p}}} .
$$

Here $N_{T ; \bar{p}}$ is the total antiproton beam intensity, $\mathcal{L}$ is the luminosity, and the factor of 2 comes from the two experiments. The $p-\bar{p}$ inelastic cross section $\Sigma_{p \bar{p}}=75 \mathrm{mb}$ [7]. At present luminosities around $4 \times 10^{31} \mathrm{~cm}^{-2} \mathrm{~s}^{-1}$, antiproton beam intensities around $700 \times 10^{9}$ particles, we find $\tau_{\mathcal{L}}(\bar{p})=49 \mathrm{~h}$. Observed antiproton beam lifetimes at the start of a store are in the range of $25-40 \mathrm{~h}$. While it is clear that the beam lifetime is mainly due to luminosity losses, other effects such as beam-beam interactions and the scattering mechanisms mentioned above have some impact. We expect that the effects of beambeam interactions will become stronger as Run II progresses with increasing intensities in both beams.

The beam-beam interactions increase the tune footprint significantly. The left plot in Fig. 16 shows the footprints due to the beam-beam interactions for bunches 1,6 , and 12 superposed on nearby sum resonances up to twelfth order. The nominal working point $\left(\nu_{x}=0.585\right.$, $\left.\nu_{y}=0.575\right)$ is chosen to lie between fifth and seventh 

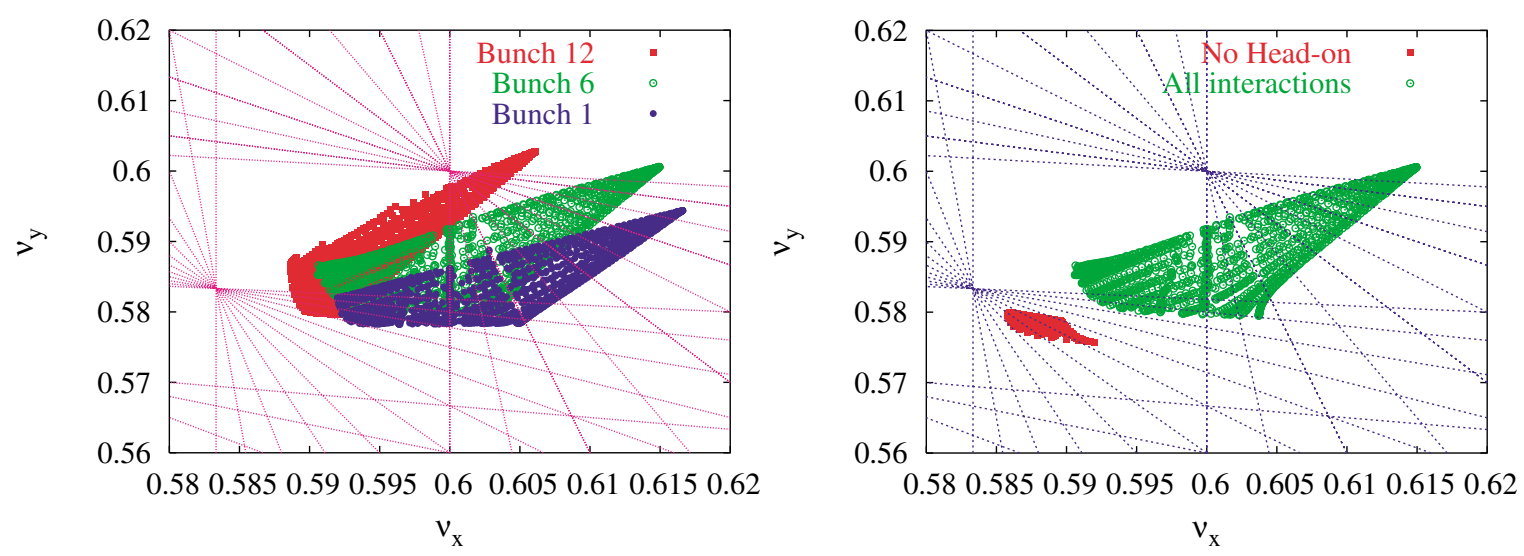

FIG. 16. (Color) Left panel: Tune footprint for bunches 1, 6, and 12 in a train at $980 \mathrm{GeV}$. Right panel: Footprint of antiproton bunch 6 with (i) all parasitic interactions but no head-on, and (ii) all the beam-beam interactions. The lattice nonlinearities are included in each case. The nearby sum resonances are shown in both plots.

order resonances. At collision, footprints of all bunches except for 1 and 12 are clustered around the footprint of bunch 6 . The major differences in the tune shifts between bunch 6 , and bunches 1 and 12 are due to the missed parasitic collision closest to the IP, upstream for bunch 1 and downstream for bunch 12 . The vertical separations at the first parasitic collision upstream of the IP are smaller than the horizontal separations. These collisions therefore contribute more to the vertical tune shift. Since these collisions are missing for bunch 1 , the vertical tune shift will be smaller for this bunch. The optics is antisymmetric about the IP, thus the horizontal separations are smaller at the 1st parasitic downstream and therefore bunch 12 has a smaller horizontal tune shift. The tune spread for all bunches is largely due to the head-on interactions. The right plot in Fig. 16 shows the footprints for bunch 6 with and without the head-on interactions. The tune spread from only the parasitic interactions is small compared to the spread from the head-on interactions and in fact has a negative sign at large amplitudes as expected from the analysis in Sec. III. We also saw in Sec. III that the chromaticity and coupling footprints on the other hand are completely due to the parasitic interactions.

\section{Dynamic aperture at collision}

We have calculated the DA of antiprotons in several situations to investigate the relative importance of the different beam-beam interactions. Figure 17 shows the six-dimensional DA after $10^{5}$ turns as a function of the initial angle in $(x, y)$ space. Zero angle corresponds to a purely horizontal amplitude while a $90^{\circ}$ angle corresponds to a purely vertical amplitude. Three cases are shown: no beam-beam interactions (single beam), headon interactions only, and all beam-beam interactions. It is clear that the head-on interactions do not change the DA much compared to the single beam case. When the para- sitics are included, there is a sharp drop in DA ranging from $3-7 \sigma$ at all angles.

In order to isolate the effect of the parasitic interactions, we consider special cases that can be considered only in simulations. We dropped the head-on interactions but included different combinations of parasitic interactions. Figure 18 compares the DA from all beam-beam interactions with two of these special cases: (i) all the parasitics and (ii) only the four parasitics nearest the IPs. We observe that the DA with all the parasitics (filled squares) is within $\pm 1 \sigma$ of the DA with all interactions (crosses) at most angles. The DA averaged over all angles is virtually the same (see Table II). The DA with only the four nearest parasitics (open squares) is significantly larger than the others for angles between $45^{\circ}-70^{\circ}$ but is close everywhere else. On average the DA with only the nearest parasitics is only about $1 \sigma$ larger. Thus of the 70

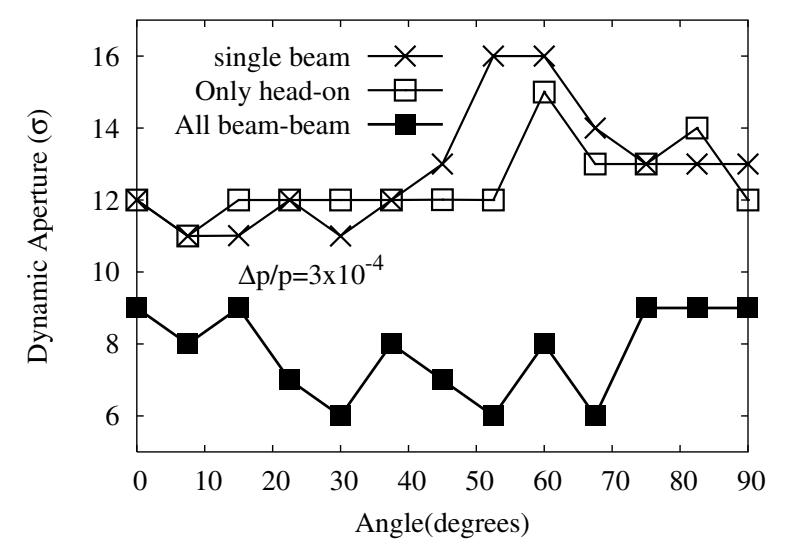

FIG. 17. Comparison of the DA with and without beam-beam interactions. DA of antiproton bunch 6 after 100000 turns as a function of angle in transverse coordinate space for three cases: (1) single beam, (2) machine nonlinearities and only the headon beam-beam interactions, and (3) machine nonlinearities and all beam-beam interactions. 


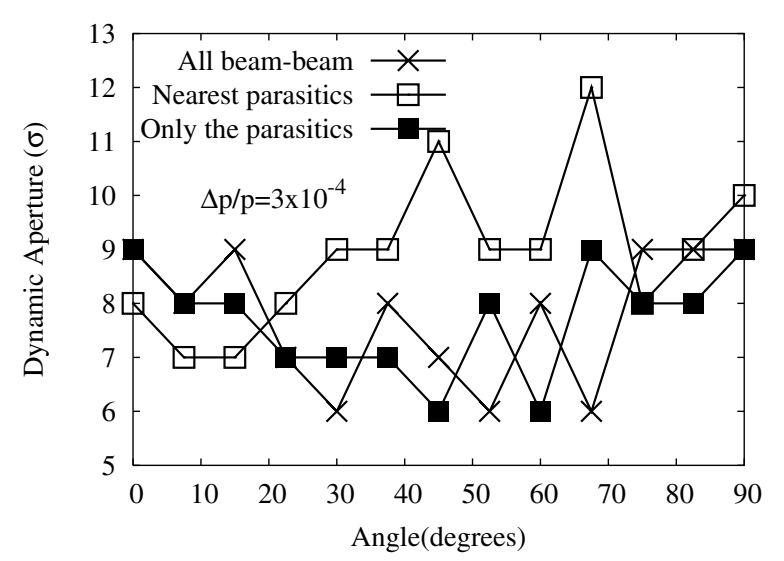

FIG. 18. Comparison of DA with different beam-beam interactions. DA of antiproton bunch 6 after 100000 turns: (1) all beam-beam interactions, (2) head-on and nearest parasitics, and (3) all the parasitics but no head-on interactions.

parasitics, the four parasitics nearest the IPs are the most important in determining the DA. Calculations at several other tunes [22] lead to the same conclusion. Table II summarizes the results of the DA calculations at the nominal working point.

The long time scale associated with the long-range interactions is evident in these results. While the DA with only the machine errors changes relatively little from $10^{5}$ turns to $10^{6}$ turns, the DA with the long-range interactions drops by another $\sim 2 \sigma$. This can be interpreted as evidence of the fact that the long-range interactions fall off with distance. It takes a relatively long time for particles to be transported to large enough amplitudes that the machine nonlinearities can take over and transport these particles out of the vacuum chamber.

Synchrobetatron resonances driven by the beam-beam interactions are found to have a strong influence on particle stability. Dynamic aperture calculations [22] with constant momentum offsets of $\Delta p / p=3 \times 10^{-4}$ and with synchrotron oscillations of the same amplitude showed that the DA in the latter case was smaller by $\sim 2 \sigma$. The effects of these resonances can be mitigated by operating with lower chromaticities and with beams of lower energy spread than at present.

\section{Scaling with proton intensity}

Figure 19 shows the dependence of the DAs on proton bunch intensities for bunches 1,6 , and 12. The DA of bunch 1 is always better than those of bunch 6; the DA of bunch 12 is also better than those of bunch 6 except at the region of intensity lower than $2 \times 10^{9}$. These results predict that the DA will drop very slowly with the proton intensity, roughly as $N_{p}^{-1 / 4}$ for most bunches. Assuming that the motion due to the nonlinearities is diffusive, the diffusive lifetime scales as $\tau_{D} \propto(D A)^{2} \sim N_{p}^{-1 / 2}$. The antiproton lifetime from luminosity falls faster, as $N_{p}^{-1}$ with increasing proton intensities. The lifetime at collision now is dominated by these $p-\bar{p}$ interactions and it is likely to remain true at design proton intensities.

TABLE II. The average and minimum 6D dynamic aperture with various configurations of beam-beam interactions. Proton bunch intensities $=2.7 \times 10^{11}$. The dynamic aperture of bunch 6 with only the parasitic collisions (PCs) is nearly the same as that with all the beambeam interactions. The nearest PCs are the parasitic interactions closest to the head-on collisions at B0 and D0. The last three rows compare the dynamic apertures of bunches 1, 6 , and 12 . The differences are insignificant.

\begin{tabular}{|c|c|c|}
\hline \multicolumn{3}{|c|}{$\begin{array}{c}\text { Bunch 6: } \nu_{x}=0.585, \nu_{y}=0.575 \\
\text { DA after } 10^{5} \text { turns }\end{array}$} \\
\hline Machine errors & $(15.2,13.0)$ & $(12.9,11.0)$ \\
\hline Head-on and machine errors & $(14.5,12.0)$ & $(12.5,11.0)$ \\
\hline Head-on, nearest PCs, machine errors & $(10.5,9.0)$ & $(8.9,7.0)$ \\
\hline Head-on, nearest PCs at $10 \sigma$, machine errors & $(13.5,12.0)$ & $(10.2,8.0)$ \\
\hline Only the parasitics, machine errors & $(10.2,9.0)$ & $(7.7,6.0)$ \\
\hline All beam-beam, machine errors & $(10.0,9.0)$ & $(7.7,6.0)$ \\
\hline \multicolumn{3}{|c|}{$\left(\langle\mathrm{DA}\rangle, \mathrm{DA}_{\min }\right)$ for bunches 1,6 and $12\left(6 \mathrm{D}, \Delta p / p=3 \times 10^{-4}\right)$} \\
\hline & $10^{5}$ turns & $10^{6}$ turns \\
\hline Single beam & $(12.9,11.0)$ & $(12.3,11.0)$ \\
\hline Bunch 1: all beam-beam & $(7.8,6.0)$ & $(5.6,3.0)$ \\
\hline Bunch 6: all beam-beam & $(7.7,6.0)$ & $(5.4,4.0)$ \\
\hline Bunch 12: all beam-beam & $(7.9,6.0)$ & $(5.8,4.0)$ \\
\hline
\end{tabular}




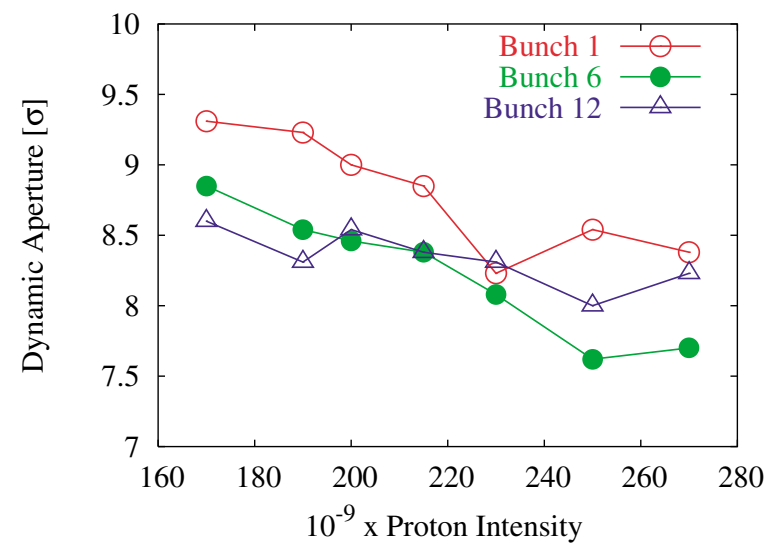

FIG. 19. (Color) Dynamic aperture of antiproton bunches 1, 6, and 12 after $10^{5}$ turns as a function of proton beam intensity at $980 \mathrm{GeV}$.

\section{Diffusion coefficients at collision}

We have seen that diffusion coefficients give useful insight into the relative importance of individual interactions and the nonlinear motion. Figure 20 shows the diffusion coefficients from individual beam-beam interactions at collision for antiproton bunch 6 . These coefficients are calculated at a $5 \sigma$ amplitude and by tracking for $10^{6}$ turns. First we observe that the diffusion from the head-on collisions is smaller than the smallest values in this figure and is therefore not shown. This also reinforces the conclusion from the dynamic aperture calculations that the head-on interactions do not lead to significant amplitude growth of particles under the conditions of the simulations. The large diffusion coefficients in this figure are due to the parasitics next to B0 and D0 where the separations are less than $6 \sigma$. In general we observe that the diffusion coefficients fall off with increasing separation. From this calculation we conclude that the nearest parasitics and a couple of others are the important ones.

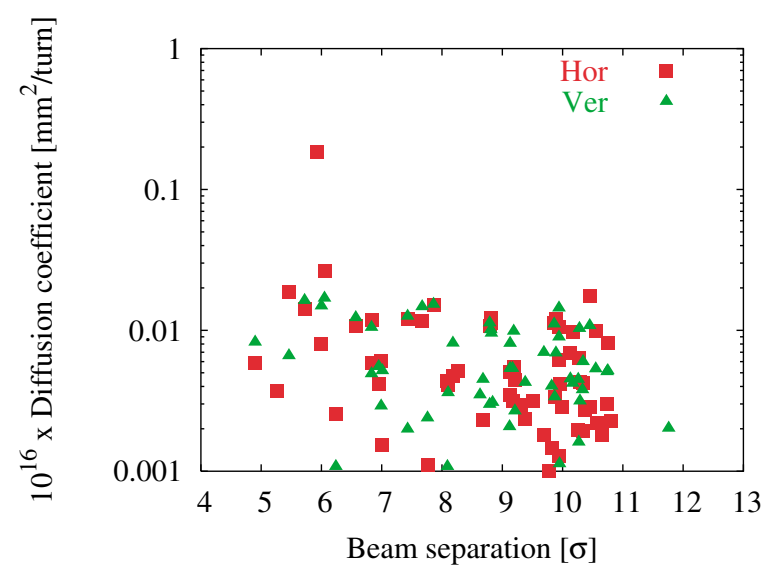

FIG. 20. (Color) Diffusion coefficients for antiproton bunch 6 at collision $(980 \mathrm{GeV})$ from individual parasitics plotted as a function of the separation at the parasitic.
At higher beam intensities, the effects due to the nearest parasitics could be mitigated by increasing the separations there. One way is to place additional separators [23] in the regions adjacent to the IRs. Other possibilities include introducing a small crossing angle $(<50 \mu \mathrm{rad})$ or changing the bunch spacing. It is also feasible to increase the helix size at collision by about $15 \%$ everywhere around the ring by increasing the voltages on the separators from their present values.

\section{CONCLUSIONS}

The effects of beam-beam interactions depend on a number of key parameters including the beam separation (in units of the rms beam size), plane of the orbits, dispersion and beta functions at the interactions, phase advances between the interactions, etc. We considered the interactions of elliptical Gaussian beams and derived theoretical expressions for important optical quantities such as amplitude dependent tune shifts, coupling, chromaticities, and resonance driving terms. These expressions allow rapid numerical evaluation. For round beams these expressions simplify and show explicitly how beam-beam tune shifts, coupling, and chromaticities depend on parameters such as the beam separations and angle of the plane of the orbit.

We have evaluated these quantities for individual antiproton bunches in the Tevatron at injection and collision. At injection we find that beam-beam chromaticities and resonance driving terms are large. Tune shifts and coupling are not significant. Beam-beam effects are smaller towards the end of a train of 12 bunches - this agrees qualitatively with observations. At collision the tune shifts are large and primarily due to the head-on collisions at $\mathrm{B} 0$ and $\mathrm{D} 0$. The next largest tune shifts are due to the parasitics on either side of B0 and D0. As a consequence the first and last bunch in the train experience very different tune shifts from all the others. This prediction has recently been confirmed by measurements. Chromaticities and resonance strengths from beam-beam interactions are significant, as at injection, but they suggest that the bunch pattern of beam-beam effects is more mixed. The resonance strengths from long-range interactions are found to be orders of magnitude larger in the tails than in the core. The overall influence of beam-beam effects on antiproton lifetimes at collision is weak; however, inelastic $p-\bar{p}$ interactions at $\mathrm{B} 0$ and $\mathrm{D} 0$ are the dominant sources of beam loss.

We also reported on numerical simulations of dynamic aperture and diffusion coefficients. Both at injection and collision we found that there is no direct connection between the dynamic aperture and the size of the tune footprint. At injection the tune footprint is largely determined by the machine nonlinearities but the dynamic aperture is determined by the long-range interactions. Experimental observation of the dynamic aperture 
when the Tevatron was operated at high chromaticities is in good agreement with the calculated values. Calculations of diffusion coefficients from individual parasitic interactions enabled us to identify the most important parasitics. As expected, we find that the diffusion coefficients with all beam-beam interactions increase much faster than linearly with the number of interactions. At collision the head-on collisions are largely responsible for the large tune footprint but they have almost no impact on the dynamic aperture. Again, the long-range interactions are found to determine the dynamic aperture. Synchrobetatron resonances are found to be important due to the machine and beam-beam induced chromaticities. Bunches with smaller momentum spread would help to reduce their effects. Diffusion coefficients have also helped us to identify the parasitics in the vicinity of the IRs as the important parasitics at collision. If beam-beam compensation turns out to be required at higher intensities, then these few parasitics would be good candidates for compensation.

\section{ACKNOWLEDGMENTS}

We thank Y. Alexahin, J. Johnstone, P. Lebrun, R. Moore, V. Shiltsev, M. Syphers, and X. L. Zhang for useful discussions, as well as J. Qiang (LBNL) and A. Kabel (SLAC) for their work on lifetime codes for the Tevatron.

[1] Proceedings of a Workshop on Beam-Beam Effects in Large Hadron Colliders, edited by J. Poole and F. Zimmermann (CERN Report No. CERN-SL/99-39 AP, 1999).

[2] Proceedings of a Workshop on Beam-Beam Effects in Circular Colliders, edited by T. Sen and M. Xiao (FNAL Report No. FNAL-Conf-01/390-T, 2001).

[3] Proceedings of the Beam Halo and Beam-Beam Workshop at BNL, edited by J. Wei, W. Fischer, and
P. Manning, AIP Conf. Proc. No. 693 (AIP, New York, 2003).

[4] T. Sen, in Proceedings of the PAC 2003, Portland, OR (IEEE, Piscataway, NJ, 2003), p. 34.

[5] X. L. Zhang et al., in Proceedings of the PAC 2003, Portland, OR (Ref. [4]), p. 1757.

[6] RUN II Handbook, http://www-bd.fnal.gov/runII/

[7] Handbook of Accelerator Physics and Engineering, edited by A.W. Chao and M. Tigner (World Scientific Press, Singapore, 1998).

[8] D. Neuffer and S. Peggs, SSC Central Design Group Technical Report No. SSC-63, 1986.

[9] Y. Alexahin, Fermilab Report No. FNAL-PUB-00-120T, 2000.

[10] G. N. Watson, A Treatise on the Theory of Bessel Functions (Cambridge University Press, Cambridge, England, 1966).

[11] B. Erdelyi and T. Sen, Fermilab Report No. FNAL-TM2171, 2002.

[12] R. J. Pasquinelli et al., in Proceedings of the PAC 2003, Portland, OR (Ref. [4]), p. 3068.

[13] A. Hofmann and S. Myers, LEP Note No. 604, 1988; K. Hirata, Nucl. Instrum. Methods Phys. Res., Sect. A 269, 7 (1988).

[14] Computer code MATHEMATICA, http://www.wolfram.com

[15] Computer code MAD, http://wwwslap.cern.ch/mad/

[16] Computer code SIXTRACK, http://frs.home.cern.ch/frs/

[17] J. Qiang et al., in Proceedings of the PAC 2003, Portland, OR (Ref. [4]), p. 3401.

[18] A. Kabel et al., in Proceedings of the PAC 2003, Portland, OR (Ref. [4]), p. 3542.

[19] T. Sen, Fermilab Report No. FNAL-PUB-00-093-T, 2000.

[20] S. Krishnagopal and R. Siemann, Phys. Rev. D 41, 2312 (1990).

[21] Y. Papaphilippou and F. Zimmermann, Phys. Rev. ST Accel. Beams 5, 074001 (2002)

[22] T. Sen and M. Xiao, FNAL Report No. Beams-doc_217, 2002.

[23] J. Johnstone, "Beam Separation with the Addition of IR Mini Separators" (unpublished). 